\title{
LegoBody: facile generation of bispecific and multi-specific antibodies
}

Shanshan Lang, Su Yang, Scott Bidlingmaier, Nam-Kyung Lee, Bin Liu ${ }^{\#}$

Department of Anesthesia, UCSF Helen Diller Family Comprehensive Cancer Center, University of California at San Francisco, San Francisco, California 94110-1305.

\#Correspondence:

Bin Liu, Ph.D., Department of Anesthesia, 1001 Potrero Ave., 1305, San Francisco, CA 94110. Tel: 415206 6973. Email: bin.liu@ucsf.edu

Key words: Bispecific, Multi-specific, linker-enforced chain pairing, protease-aided linker removal, thrombin, LegoBody 


\begin{abstract}
Bispecific and multi-specific antibodies are capable of recognizing multiple ligands simultaneously or synergistically, creating complex biological interactions not achievable by monoclonal antibodies, thus expanding opportunities for novel therapy development. With the large number of monoclonal antibodies either approved or under clinical development, there are numerous opportunities to combine their specificities to further improve therapeutic potential. Although simple in concept, clinical development of biand multi-specific antibodies face several challenges, chief of which is how to efficiently and reliably produce bispecific and multi-specific antibodies with expected specificity and desired biophysical properties. In this study, we developed a modular approach that uses temporary linkers to enforce proper chain pairing and proteases such as thrombin to remove those linkers from the final product. Combined with the 'knob-into-hole' design, we can generate IgG-like, bi- or multi-specific antibodies from any pre-existing monoclonal antibodies. The approach is highly versatile and applicable to any monoclonal antibody pair or panel, expediting evaluation and therapeutic development of bi- and multi-specific antibodies.
\end{abstract}




\section{Introduction}

Antibodies with bi- or multi-specificity hold great promise for the next generation of therapeutic drugs against a variety of diseases, including cancers, infections, and immunological disorders. Compared to traditional monoclonal antibodies that specifically recognize one ligand, bi- or multi-specific antibodies can recognize two or more ligands and thus may provide an advantage in co-engaging different cell types ${ }^{1-4}$, creating synthetic specificity ${ }^{5}$, altering internalization dynamics ${ }^{6,7}$, synergistically neutralizing virus and toxin ${ }^{8-10}$, and simultaneously block multiple signaling pathways to maximize therapeutic benefits ${ }^{11-17}$. In addition, bi- and multi-specific antibodies can increase sensitivity and breath for recognizing target cells, tissues, or pathogens ${ }^{8,18-20}$. Some bispecific antibodies were also designed to possess desirable properties other than recognition, such as enhanced production, extended half-time, or increased tissue penetration $^{21,22}$.

Production of bi- or multi-specific antibodies is more complicated than monoclonal antibodies $^{18,23,24}$. Many different formats of bi-specific antibodies have been designed, including chemical conjugation of two disparate antibodies, tandem single-chain variable fragments $(\mathrm{scFv})$ or Fab domains, and $\mathrm{scFv}$ or Fab fusion to immunoglobulins or other scaffold proteins ${ }^{18,23,25-34}$. The design of multi-specific antibodies is often derived from these bispecific formats but with substantially higher complexity ${ }^{35-37}$. Each of these designs, and their derivatives, features distinct topology and thus may have non-identical biological functions and different pharmacokinetics, which needs to be tested 
experimentally. Depending on the molecular form of the building block, some formats suffer from drawbacks, ranging from poor production, low in vivo stability, and immunogenicity ${ }^{23,38}$. Consequently, new methods and formats to produce bi- or multispecific antibodies are important topics for exploration.

Among the various designs, the IgG-like format is our main area of interest due to its resemblance to natural antibody, which often have good yields, stability, and relatively low immunogenicity ${ }^{39,40}$. To produce these IgG-like molecules, two major problems have to be addressed: proper pairing of heavy chains from different antibodies, and correct pairing of the light chains to their corresponding heavy chains. The first issue is often addressed by the now classic knobs-into-holes (KIH) Fc mutants that enforce the formation of heavy chain heterodimers ${ }^{41,42}$. However, the desired heterodimer in such a design is not exclusive, with homodimers still present, likely due to insufficient thermal stability of the $\mathrm{CH} 3$ domain and the Fc interface of the KIH heterodimer ${ }^{43}$. In fact, both knob and hole Fc domains can form homodimers, causing substantial contamination in products that were generated by the KIH approach alone ${ }^{44-48}$. As the homodimer often shares similar biophysical features as the heterodimer, such as size and isoelectric point, optimization and purification of the $\mathrm{KIH}$ bispecific format can be challenging and timeconsuming. Additional modifications of the KIH Fc have been explored to enhance the formation of heterodimer ${ }^{45-49}$.

To avoid promiscuous association of light chains to heavy chains in bispecific antibody production, different antibodies (with different ligand binding specificity) using one 
common light chain have been selected from phage or yeast display libraries, providing a popular solution to the light chain pairing problem ${ }^{50-52}$. However, this restriction on light chain reduces the sequence space that can be explored for binding affinity/specificity and downstream developability. The CrossMab format is an alternative approach for solving the light chain pairing problem, swapping only one pair of the variable or constant domains in one of the Fab fragments, and thus inhibiting chain mismatching between the switched Fab and the un-switched ones ${ }^{53}$. Nonetheless, incorrect pairing of light chains are often observed, resulting in variable levels of product heterogeneity ${ }^{23}$. The issue of immunogenicity of the hybrid variable-constant domain remains to be investigated.

The feasibility of single-chain IgG ( $\mathrm{scIgG}$ ) has been demonstrated by previous studies and can be used to resolve the light chain pairing problem ${ }^{54-56}$. However, an extended linker between light and heavy chains, varying between 30-60 residues in length, was retained in the final product, limiting its utility. Here, we sought to develop an approach to produce single-chain IgG with cleavable linkers, allowing for removal of the undesired linkers in vitro through the use of proteases. We successfully introduced a thrombin cleavable linker between the light chain and the heavy chain, which can be removed by commercially available enzymes with high efficiency and accuracy. Antibodies produced using this approach show similar yield as the original antibodies, feature intact ligandbinding affinity, and contain only a few linker residues. Compared to an in vivo cleavage system designed in a previous study ${ }^{57}$, the cleavage in our system happens posttranslationally and post-purification, ensuring correct pairing of the chains during cleavage. Furthermore, the linkers enable us to engineer extra features in each of the two 
chains, such as different affinity tags, which can be used for easy purification of highly pure bispecific IgG molecules. Finally, unlike an approach that is designed to produce the entire bispecific IgG-like molecule as a single polypeptide before enzymatic processing ${ }^{58}$, our approach is totally modular, demands far less on the protein synthesis machinery, and allows facile production of not only bispecific but also multi-specific antibodies, with IgG-like backbone and Fab-based binding modules. 


\section{Results}

Monoclonal antibodies produced from thrombin-removable single-chain IgGs show similar biochemical features as native IgGs

Single-chain IgGs using peptide linkers to join the light chain and heavy chain have been investigated previously for production and ligand binding ${ }^{54-56}$. The linker in scIgG remains a liability for therapeutic development. We therefore developed an efficient system to remove the peptide linker to obtain a true IgG-like molecule.

We tested several commercially available proteases and determined that thrombin is the most suitable enzyme because of its efficiency, accuracy, and compatibility with nonreducing environments. We chose the clinically established antibodies, Ipilimumab (Ipili) and Daratumumab (Dara) as the study antibodies and generated scIgGs of Ipili and Dara with a linker in-between the light chain and heavy chain. The linker, sc36TMB, is a flexible 36 -residue peptide adapted from a previous study ${ }^{55}$, with additional thrombin cleavage sites on both the N- and C-terminus (Fig.1A, Supplemental Fig. S1A).

The engineered scIgG displayed similar yield as the original antibodies (Supplemental Fig. S1B). Furthermore, the linker was successfully removed by thrombin cleavage, yielding a $\sim 150 \mathrm{kDa}$ antibody product formed by disulfide-bonded light ( $25 \mathrm{kDa})$ and heavy chains $(\sim 50 \mathrm{kDa})$, same as the natural antibody (Fig. 1B). Following thrombin cleavage, a 5-mer peptide (-GLVPR) remained at the C-terminus of the light chain, as 
well as two residues (-GS) at the N-terminus of the heavy chain (Supplemental Fig. S1A). To evaluate if the processed IgG molecule possesses an intact paratope, we tested ligand binding by ELISA (Fig. 1C, D). Ipilimumab binds to the CTLA4-Fc with an $\mathrm{EC}_{50}$ of approximately $0.99 \pm 0.13 \mathrm{nM}$, while the $\mathrm{EC}_{50}$ of the cleaved Ipili-sc36TMB is estimated to be about $0.83 \pm 0.11 \mathrm{nM}$ (Fig. 1C). Likewise, Daratumumab binds its ligand, CD38, with $\mathrm{EC}_{50}$ of approximately $1.56 \pm 0.11 \mathrm{nM}$, and the cleaved Dara-sc36TMB has an $\mathrm{EC}_{50}$ of $1.24 \pm 0.09 \mathrm{nM}$ (Fig. 1D). The similar $\mathrm{EC}_{50}$ values between the processed scIgGs and the original antibodies indicate that the engineered linker and the cleavage process do not interfere with the formation of the antibody or the integrity of its paratope.

\section{Bispecific antibodies produced from thrombin-cleavable single-chain IgGs with the}

\section{'knobs-into-holes' Fc heterodimer}

We next expand the study from monoclonal to bispecific antibody generation. The KIH design features critically paired mutations in the 'knob' Fc (T366W) and 'hole' Fc (T366S/L368A/Y407V), which enforces pairing of the KIH Fc heterodimer ${ }^{41,42}$. However, this KIH design does not enforce proper light chain pairing.

The successful production and thrombin-cleavage of the scIgGs to IgG molecules provides a plausible solution for enforcing correct pairing between light and heavy chains. Thus, we sought to generate bispecific antibodies using the KIH Fc system in conjunction with a thrombin-removable linker. We chose two sets of antibodies, Ipilimumab pairing with Daratumumab, and Ipilimumab paring with Herceptin, as the test samples. Our scheme to produce bispecific antibodies is shown in Fig. 2A. In brief, the light chain of 
each antibody is fused with the corresponding heavy chain via the thrombin-removable linker. The Fc used for Ipilimumab is the 'hole' Fc, while the Fc for either Daratumumab or Herceptin is the 'knob' Fc. Bispecific antibodies were produced by co-transfecting HEK293A cells with the 'knob' and 'hole' scIgG constructs at a 1:1 ratio, and purified from culture supernatant by protein A agarose, and processed by thrombin to remove the linker.

Using this system, we were able to obtain relatively pure IgG-like molecules for both bispecific antibodies with estimated molecular weights of $\sim 150 \mathrm{kDa}$ under non-reducing condition (Fig. 2B). The KIH product can be separated into light chain $(\sim 25 \mathrm{kDa})$ and heavy chain $(\sim 50 \mathrm{kDa})$ by reduction with $\beta$-mercaptoethanol ( $\beta$-ME) (Fig. 2B). We assessed the purity of the $\mathrm{KIH}$ product using analytical hydrophobic interaction chromatography (HIC). Both Ipili-Dara-KIH and Ipili-Her-KIH display a dominant bispecific component, comprising $71 \%$ and $73 \%$ of the total product, respectively (Fig. S2, S3).

We also investigated the integrity of the paratopes of each Fab in the bispecific by ELISA. Unlike an IgG molecule that binds bivalently to one ligand, the binding of the IgG-like bispecific to each of its two ligands is monovalent. To minimize the influence of valency in the ELISA assay, we mobilized antibodies on plates and assessed binding to ligands in solution. As shown in Fig. 2C \& D, and Supplemental Fig. S4A, the Ipili-Dara-KIH is capable of binding to both CTLA4 and CD38, in manners that are similar to the parental monoclonal antibodies (Ipilimumab and Daratumumab), indicating that the bispecific 
antibodies possess intact paratopes. Similar results were observed for the bispecific IpiliHer-KIH (Fig. 2E \& F, and Supplemental Fig. S4B).

\section{Use of linker-embedded affinity tags for further purification of bispecific antibodies}

Contamination in bispecific antibodies produced by the KIH approach has been observed previously ${ }^{45-47,49}$, which primarily arises from 'hole-hole' or 'knob-knob' homodimers. Common procedures for antibody purification, such as protein A or $G$ affinity chromatography, reply on recognition of the Fc domain and thus cannot effectively distinguish the bispecific $\mathrm{KIH}$ heterodimer from contaminating homodimers. Prior approaches to enhance the efficiency of correct pairing of the KIH heterodimer or eliminate the non-bispecific contamination often involve introducing additional mutations and additional steps in purification.

Here, we took advantage of the removable nature of the linker and embedded a pair of affinity tags, the 10-His tag and Twin-Strep-Tag ${ }^{\circledR}$, into the linkers to purify the bispecific product (Fig. 3A). The KIH heterodimer would possess both affinity tags and thus be readily distinguished from homodimers. We constructed plasmids expressing Ipilimumab with a Twin-Strep-Tag ${ }^{\circledR}$ linker and the 'hole' Fc mutant, and Daratumumab or Herceptin with a 10-His tag linker and the 'knob' Fc mutant, co-transfected HEK293A cells to generate bispecific antibodies, i.e., Ipili-Dara-KIH and Ipili-Her-KIH. The supernatant was sequentially purified using Ni-NTA and Strep-Tactin ${ }^{\circledR} \mathrm{XT}$ resin to obtain antibody products with both tags and thus both specificities. The linkers were removed by thrombin cleavage to generate the final product. SDS-PAGE shows that bispecific 
antibodies produced in this manner contain substantially lower amounts of containments (labeled with '*') (Fig. 3B). HIC-HPLC analysis showed that the purities of the IpiliDara-KIH and Ipili-Her-KIH are nearly $100 \%$ and about 92\%, respectively (Fig. 3C, D).

We next studied how bispecific antibodies recognized cells expressing target antigens. The Jurkat cell line has been used to study CD38 binding ${ }^{59,60}$. We found that indeed Jurkat cells express CD38 and are bound by Daratumumab (Fig. 3E). However, little to no expression of CTLA4 was detected by Ipilimumab (Fig. 3E). Thus Jurkat cells are useful mainly for assessing the CD38 binding arm. The bispecific antibody, Ipili-Dara$\mathrm{KIH}$, which is monovalent in each specificity, binds to Jurkat cells with $\mathrm{EC}_{50}$ of $2.26 \pm$ $0.26 \mathrm{nM}$ (Supplemental Fig. S5A). Daratumumab, a bivalent IgG, binds to Jurkat cells with $\mathrm{EC}_{50}$ of $0.31 \pm 0.05 \mathrm{nM}$. The bispecific Ipili-Dara-KIH binds to Jurkat cells with a higher median fluorescence intensity (MFI) value compared with Daratumumab, consistent with its monovalent binding mode.

Similarly, we evaluated how the bispecific Ipili-Her-KIH antibody binds to the breast cancer cell line MCF7 that has been reported to express both ErbB2 (Her2) and CTLA4 61. We observed a high staining signal by flow cytometry on MCF7 by Herceptin, but a rather low signal by Ipilimumab. Thus we used MCF7 cells to evaluate the Her2-binding arm only. The bispecific Ipili-Her-KIH binds to MCF7 (Fig. 3F) with $\mathrm{EC}_{50}$ of $1.39 \pm 0.21$ nM (Supplemental Fig. S5A). Herceptin, a bivalent IgG, binds to MCF7 (Fig. 3F) with $\mathrm{EC}_{50}$ of $0.60 \pm 0.18 \mathrm{nM}$ (Supplemental Fig. S5A). The total MFI for the bispecific IpiliHer-KIH is higher than Herceptin, consistent with its monovalent binding mode. 
To evaluate binding of bispecific antibodies to CTLA4 on cell surface, HEK293A cells were transiently transfected with a construct expressing the human CTLA4 gene. After culturing for 18 hours, the cells were harvest and analyzed by flow cytometry for binding by Ipilimumab IgG, Ipilimumab Fab, Ipili-Dara-KIH, and Ipili-Her-KIH. Like Ipilimumab, both bispecific antibodies were able to bind to the CTLA4-transfected cells (Supplemental Fig. S5B), confirming that the anti-CTLA-4 arm is functional. The apparent binding affinities of the bispecific antibodies are lower than that of the bivalent Ipilimumab IgG, an expected result given the valency difference, but higher than the Ipilimumab Fab that is also monovalent (Supplemental Fig. S5C). MFI values at saturation binding are higher for the bispecific (and the Ipilimumab Fab) than the Ipilimumab IgG, again consistent with the monovalent vs. bivalent binding mode.

\section{Facile generation of multi-specific antibodies using thrombin-removable linkers}

The generation and application of antibodies with even higher order of specificity and complexity are challenging tasks that have only been attempted a limited number of times 35-37. In general, as the complexity of the molecule grows, the difficulty grows disproportionally or exponentially in proper assembly of six, eight, or more chains into one antibody. Here, we take advantage of the modular nature of the linker-enforced chain assembly and generate multi-specific antibodies using the enzymatically cleavable linker. In simplified terms, these molecules were designed based on the IgG-like bispecific with additional specificities introduced by appending Fab domains to either the N-terminus (Fig. 4 \& 5, tandem Fabs) or C-terminus (Fig. 6) of the bispecific molecule. 
In the case of tandem Fab constructs shown in Fig. 4 (tri-specific) and 5 (tetra-specific), the four different chains (two heavy and two light chains) of two antibodies (I, III) are genetically fused into one polypeptide in the following configuration: light chain-sc36TMB linker-heavy chain-N-linker-light chain (I)-sc36TMB linker-heavy chain (I) and 'hole' Fc mutant (shown in Fig. 4A). This tandem Fab construct is paired with one antibody construct (position II) with the 'knob' Fc mutant to produce the trispecific antibodies (Fig. 4A). To produce the tetra-specific molecule, another two antibodies (II, IV) were constructed in a second tandem Fab construct with the 'knob' mutant in a configuration similar to that of the 'hole' Fc mutant (Fig. 5A).

Co-expression of corresponding constructs produces the precursors for either tri-specific (Fig. 4A) or tetra-specific (Fig. 5A) molecules, which are processed by thrombin cleavage to remove the linker scaffold within each Fab unit. In this way, we were able to generate the asymmetric tri-specific antibody (Tri-N-Fabs) and the symmetric tetraspecific antibody (Tetra-N-Fabs), with Ipilimumab in position I, Daratumumab in position II, Herceptin in position III, and Atezolizumab in position IV (in the case of Tetra-N-Fabs).

Purified Tri-N-Fabs and Tetra-N-Fabs displayed correct molecular weights by SDSPAGE analysis, estimated to be $200 \mathrm{kDa}$ and $250 \mathrm{kDa}$ respectively, under non-reducing conditions and were separated into polypeptides with molecular weights of approximately $50 \mathrm{kDa}$ and $25 \mathrm{kDa}$ under reducing conditions (Fig. 4B \& 5B). Purity of the final products was assessed by analytical HIC-HPLC and found to be approximately $87 \%$ for 
Tri-N-Fabs (Fig. 4C) and 95\% for Tetra-N-Fabs (Fig. 5C). We evaluated antigen binding by ELISA, where the antibody was immobilized on plate, and tested for binding to biotinylated ligands in solution (CTLA4-Fc, CD38, ErbB2-Fc, and PD-L1). Both the TriN-Fabs and the Tetra-N-Fabs showed binding to all corresponding ligands with apparent affinity similar to that of the parental antibodies (Fig. 4D-F and Supplemental Fig. S7A for Tri-N-Fabs; Fig. \& 5D-G and Supplemental Fig. S7B for Tetra-N-Fabs). These results indicate that the Tri-N-Fabs and the Tetra-N-Fabs possess the designed paratopes that are functional.

An alternative way to arrange the different Fab modules in a tri- or tetra-specific antibody is to append the extra Fabs to the C-terminus of the Fc domain (Fig. 6A for the trispecific, Tri-C-Fabs; and Fig. 6B for the tetra-specific, Tetra-C-Fabs). For the asymmetric Tri-C-Fabs, the two chains are in the following configuration: light chain (I)-sc36TMB linker-Fab heavy chain (I)-'hole' Fc mutant-C-linker-light chain (III)-sc36TMB linker-Fab heavy chain (III); and light chain (II)-sc36TMB linker-Fab heavy chain (II)-'knob' Fc mutant (Fig 6A). The C-linker was designed as a 13-residue long Gly-Ser peptide. For the symmetric Tetra-C-Fabs, light chain (I/II)-sc36TMB linker-Fab heavy chain (I/II)-'hole'/'knob' Fc mutant-C-linker-light chain (III/IV)-sc36TMB linker-Fab heavy chain (III/IV) (Fig. 6B). These multi-specific antibodies were produced by co-transfecting plasmids expressing the corresponding chains followed by purification on protein A agarose. The linkers in-between light and heavy chains were removed by thrombin cleavage prior to analysis. 
We successfully produced a tri-specific antibody (Tri-C-Fabs) with Ipilimumab Fab at position I, Daratumumab Fab at II, and Herceptin Fab at III; and a tetra-specific antibody (Tetra-C-Fabs) with the addition of Atezolizumab Fab at IV. The main products for both Tri-C-Fabs and Tetra-C-Fabs displayed the correct molecular weights by SDS-PAGE analysis (Fig. 6C). Analytical HIC-HPLC indicated that the overall purities of the Tri-CFabs and Tetra-C-Fabs were approximately 93\% and 79\%, respectively (Supplemental Fig. S6A, B). Both Tri-C-Fabs and Tetra-C-Fabs were able to bind their intended ligands by ELISA, with $\mathrm{EC}_{50}$ similar to that of the parental antibodies, i.e., Ipilimumab, Daratumumab, and Atezolizumab (Fig. 6D-J, Supplemental Fig. S7C, D). We observed a reduction in target binding by the Herceptin Fab in these multi-specific products. With regard to ErbB2 binding, the Tri-C-Fabs and Tetra-C-Fabs display approximately 9- and 3- fold higher $\mathrm{EC}_{50}$ values compared to the parental antibody Herceptin. However, the effect seems to be unique to the Herceptin Fab as the Atezolizumab Fab in a similar position in Tetra-C-Fabs was not affected (Fig. 6J).

We also evaluated the binding kinetics of the bispecific or multi-specific antibodies to corresponding ligands by biolayer-interferometry (BLI). We immobilized the biotinylated ligands on the streptavidin-coated sensor and measured antibody binding. We found that the bispecific and multi-specific antibodies bind to their ligands in manners expected from their monovalent binding mode (Fig. S8). The parent monoclonal IgGs showed lower $\mathrm{K}_{\mathrm{d}}$ values due to avidity (bivalent vs. monovalent), but the parent monovalent Fab (Ipilimumab Fab) showed similar $\mathrm{K}_{\mathrm{d}}$ as the bipsecific and multi-specific antibodies. 


\section{Discussion}

In this study, we have developed a facile and modular platform to generate bispecific and multi-specific antibodies from any pre-existing monoclonal antibody. The use of thrombin-removable linkers enforces correct pairing of light and heavy chains and enables efficient in vitro removal of these linkers following purification. Comparing other bispecific formats, such as the CrossMAb or common light chain IgG molecules, our format requires minimal efforts in design and optimization, imposes no restrictions on the underlying monoclonal antibody (the building block), and can be used as tools to readily generate and evaluate bispecific antibodies from any two monoclonal antibodies of interest. The linker we introduced to enforce proper chain paring is very malleable and can be used to introduce versatile features to aid purification. As a proof of concept, we have designed different affinity tags into the linker, and demonstrated that sequential purification by affinity chromatography can effectively eliminate homodimer contaminations. Other features such as asymmetric length or charge (isoelectric point) can be incorporated into the linker to customize the purification scheme.

We further expanded the approach, which is modular in nature and permits Lego-like assembly, to multi-specific antibody generation. We generated two classes of tri-specific and tetra-specific antibodies by appending additional Fab domains to either the Nterminus of the light chain or the C-terminus of the heavy chain in the starting bispecific molecule. The added Fab domains also use thrombin-removable linkers to enforce correct heavy and light chain pairing. We have successfully generated several tri- and tetraspecific molecules and verified their binding to corresponding ligands. Given the modular 
nature of the process, our approach should be applicable to the generation of even higher order of specificities. This marks a major distinction from an approach where the IgGlike bispecific is produced as a single giant polypeptide chain before enzymatic processing ${ }^{58}$, which has production issues due to the sheer size of the molecule and is essentially limited to bispecific only. Given the explosion of information on the molecular mechanism of human diseases and the rapid expansion of the list of potential targets, multi-specific antibodies are likely to become an expanding class of novel therapeutics due to their unique ability to generate synergistic or synthetic interactions among targets, thus uncovering new biology and targeting opportunities. Our approach offers a means of facile generation of novel molecular tools to fully explore those opportunities.

\section{Materials and Methods}

Monoclonal antibody expression and purification: Genes encoding antibody variable domains were synthesized by gBlock ${ }^{\circledR}$ (Integrated DNA Technologies). Plasmids for the heavy and light chains for each antibody were separately cloned in the Abvec vector as previously described 5, 62. Antibodies were produced by co-transfecting plasmids expressing the heavy and light chains at a 1:1 ratio in HEK293A cells for 6-8 days, followed by purification of culture supernatant on protein A agarose (Pierce/Thermo Scientific). 
Monoclonal single-chain IgG expression and purification: The sc36TMB linker (GLVPRGSGSGGGSGGGSEGGGSEGGGSEGGGSEGGGSGGGSGGLVPRGS), and the heavy chain variable domain were fused by PCR reaction. The resultant DNA fragment was subcloned into Ig- $\gamma$ Abvec vector and fused with the heavy chain constant domain and Fc fragment to generate the complete single-chain IgG. The plasmid was transfected into HEK293A cells and scIgG molecules were purified from culture supernatant by protein A agarose (Pierce/Thermo Scientific).

Thrombin cleavage and removal of the enzyme: To remove the sc36TMB linker, scIgG was mixed with thrombin at the ratio of 50-100 $\mu \mathrm{g}$ antibody per unit of thrombin (Millipore, 605160), and the mixture was incubated at $37^{\circ} \mathrm{C}$ for 2 hours. The processed IgG molecule was re-purified by protein $A$ agarose to remove the enzyme. The conversion of the scIgG to IgG was verified by SDS-PAGE with reducing agent added to the sample.

Bispecific antibody expression and purification: The 'knob' $\mathrm{Fc}(\mathrm{T} 366 \mathrm{~W})$ or 'hole' $\mathrm{Fc}$ (T366S/L368A/Y407V) fragment ${ }^{41,42}$ were introduced to the Ig- $\gamma$ chain by site-directed mutagenesis. The fused gene encoding the complete light chain, sc36TMB linker, and heavy chain variable domain was subcloned into either the 'knob' or 'hole' vector as indicated in Results. To produce the scIgG bispecific antibody, a pair of the 'knob' and 'hole' vectors, each encoding one scIgG antibody, were co-transfected into HEK293A cells at a 1:1 ratio. After culturing for 6-8 days, the bispecific scIgG was purified from 
culture supernatant by protein A agarose. The sc36TMB linkers in the bispecific scIgG were removed by thrombin cleavage as described above, yielding the bispecific IgG.

Tri-specific and Tetra-specific antibody expression and purification: The AgeI site between the signal peptide and N-terminus of the antibody gene in the Abvec vector was retrained in the construct for scIgG with either the 'knob' or 'hole' Fc. This AgeI site was used to introduce an additional Fab gene (with a thrombin removable linker in-between the light and heavy chain) and the N-linker (-ASTKGPSGSG-) by ligase independent cloning ${ }^{63}$. To produce the tri- and tetra-specific antibodies, we co-transfected the pair of 'knob' and 'hole' vectorinto HEK293A cells, collected supernatant after 6-8 days which was purified on protein A agarose, and removed the sc36TMB linkers by thrombin. For producing the tri-specific antibody, the tandem Fab (Ipilimumab and Herceptin) with the 'hole' Fc vector was paired the single-chain Daratumumab vector with 'knob' Fc. For the tetra-specific, the tandem Fab (Ipilimumab and Herceptin) with the 'hole' Fc vector was paired the tandem Fab (Daratumumab and Atezolizumab) with 'knob' Fc vector. Both Tri-N-Fabs and Tetra-N-Fabs molecules were submitted for ion-exchange chromatography for additional purification.

Alternatively, a HindIII site at the C-terminus of the antibody gene was used to introduce the C-linker (-GGGSGGGSGGGSG-) and an extra Fab domain to the 'knob' or 'hole' vector. To produce the Tri-C-Fabs molecule, the 'hole' vector with two Fab modules (Ipilimumab at $\mathrm{N}$-terminus and Herceptin at C-terminus) was co-expressed with the 'knob' Fc vector for the single-chain Daratumumab Fab in HEK293A cells. To produce 
the Tetra-C-Fabs molecule, the above 'hole' vector was co-expressed with the 'knob' Fc vector with two Fab modules (Daratumumab at N-terminus and Atezolizumab at Cterminus) in HEK293A cells. Following purification by protein A agarose, the unnecessary linkers were removed by thrombin. Ion-exchange chromatography was applied to improve the purity.

Purification by ion-exchange chromatography: The tri-specific and tetra-specific molecules were further purified by ion-exchange chromatography after the removal of intra-Fab linkers. In brief, the molecules were applied to a Mono $\mathrm{S}^{\mathrm{TM}}$ 5/50 GL column (GE Healthcare) on ÄKTA (GE Healthcare). The following buffers were used: mobile phase buffer A: 20 mM MES (2-morpholin-4-ylethanesulfonic acid), pH 6.0; and mobile phase buffer B: $20 \mathrm{mM}$ MES, $1 \mathrm{M} \mathrm{NaCl}$, pH 6.0. After loading and washing the samples with buffer A, gradient elution with $10 \%$ to $50 \%$ B were used for fractionation. Fractions were collected and analyzed by SDS-PAGE.

Ligand preparation and biotinylation: Extracellular domain Fc fusions for human CTLA4 (CTLA4-Fc) and ErbB2 (ErbB2-Fc) were purchased from Abcam (ab180054 and ab168896). These ligands were biotinylated by the EZ-link ${ }^{\circledR}$ Sulfo-NHS-LC-Biotin according to the manufacturer's protocol (Thermo Scientific). Excessive biotin was removed by buffer-exchange into PBS, and biotinylated ligands were concentrated and stored at $-80^{\circ} \mathrm{C}$. The recombinant extracellular domain of CD38 and PD-L1 was produced as a 6-His and AviTag ${ }^{\mathrm{TM}}$ fusion in HEK293 cells and purified by Ni-NTA followed by in vitro biotinylation by BirA biotin ligase (Avidity). 
ELISA and $\boldsymbol{E C}_{50}$ estimation: To accommodate the differences in valency between monoclonal and bi-, tri-, or tetra-specific antibodies, we performed the ELISA assay by immobilizing the antibody on a microtiter plate and testing binding to ligands in solution. The antibodies were diluted to $1 \mu \mathrm{g} / \mathrm{ml}$ in PBS and $100 \mu \mathrm{l}$ of this solution per well were applied to the Nunc MaxiSorp ELISA plate (Thermo Fisher) for coating overnight. The plate was washed three times with PBS, blocked with 4\% BSA at RT for 1 hour, and incubated with corresponding biotinylated ligands at various concentrations at RT for 1 hour, each condition in triplicates. The plate was then washed five times with the washing buffer $(0.05 \%$ Tween-20 in PBS), incubated with $0.1 \mu \mathrm{g} / \mathrm{ml}$ HRP-conjugated streptavidin (Pierce/Thermo Fisher) at RT for 30 min, washed 3 times with the washing buffer, and incubated with the peroxidase substrate solution (SureBlue ${ }^{\circledR}$, Seracare) at RT for 3-5 min before the reaction was terminated by adding equal volume of $1 \mathrm{M} \mathrm{HCl}$. The absorbance at $450 \mathrm{~nm}$ of each well was detected by a microtiter plate reader (Synergy BioTek ${ }^{\circledR}$ instrument). The absorbance value as a function of the ligand concentrations was analyzed to obtain the $\mathrm{EC}_{50}$ value by curve fit (Prism, GraphPad).

Purity assessment by analytical hydrophobic interaction chromatography: Purified antibodies were analyzed by HIC-HPLC with the infinity 1220 LC System (Agilent). Mobile phase A consisted of $25 \mathrm{mM}$ phosphate and $1.5 \mathrm{M}$ ammonium sulfate, $\mathrm{pH} 7.0$; and mobile phase B 25\% (v/v) isopropanol in $25 \mathrm{mM}$ phosphate, $\mathrm{pH}$ 7.0. Antibody samples were loaded onto a TSKgel HIC column (Tosoh Bioscience) and operated at 0.5 
$\mathrm{mL} / \mathrm{min}$ with a gradient from $10 \%$ to $100 \%$ B. Absorbance was detected at $280 \mathrm{~nm}$. Purity was estimated by area integration using OpenLab CDS software (Agilent).

Cell binding analysis by flow cytometry: Jurkat and MCF7 cell lines were obtained from American Type Culture Collection (ATCC) and cultured in Dulbecco's Modified Eagle Medium (DMEM) with $10 \%$ fetal calf serum and $100 \mu \mathrm{g} / \mathrm{ml}$ penicillin-streptomycin in humidified atmosphere of $95 \%$ air and $5 \% \mathrm{CO}_{2}$ at $37{ }^{\circ} \mathrm{C}$. For flow cytometry analysis, approximately 50,000 cells were incubated with different monoclonal, bispecific or multi-specific antibodies (the highest concentration of $200 \mathrm{nM}$ with serial 4-fold dilutions) at RT for 1 hour, washed 3 times with PBS and further incubated with the secondary antibody solution (Alexa Fluor $647^{\circledR}$-conjugated goat anti-human IgG, final concentration of $1 \mu \mathrm{g} / \mathrm{ml}$ ) at RT for 1 hour, washed 3 times with PBS and analyzed by flow cytometry (Accuri ${ }^{\mathrm{TM}} \mathrm{C} 6, \mathrm{BD}$ Biosciences). The median fluorescence intensity (MFI) values were analyzed by Prism (GraphPad) to obtain $\mathrm{EC}_{50}$ values by curve fitting.

$K_{d}$ determination by bio-layer interferometry. A Gator instrument (Probe Life) was used to determine $\mathrm{K}_{\mathrm{d}}$ of antibody-ligand interactions by bio-layer interferometry. Biotinylated ligands were diluted to $5-10 \mu \mathrm{g} / \mathrm{ml}$ in the provided kinetics buffer (Probe Life) and immobilized onto streptavidin-coated biosensors. The sensors were sequentially incubated with antibodies $(200 \mathrm{nM})$ and kinetics buffers for 180 seconds to assess rates of association and disassociation. $\mathrm{K}_{\mathrm{d}}$ values were analyzed by the Gator software (Probe Life). 


\section{Author contributions}

S.L. and B.L designed the project and wrote the manuscript. S.L. performed overall experiments. S.Y. performed analytical HPLC and provided anti-Erbb2 and CD38 antibodies. S.B. helped with FACS experiments and provided knob and hole vectors. N.L. provided PD-L1 ligands. BL conceived the overall project idea. All authors edited the manuscript.

\section{Acknowledgement}

We thank Weihua Wen for help with protein purification. This work is supported in part by grants from the National Institutes of Health (R01 CA118919, R01 CA129491, R01 CA171315 and R01 CA223767).

\section{Declare of Interest}

The authors are inventors of a patent related to this work. 


\section{Reference}

1. Schetelig J, Link CS, Stuhler G, Wagner EM, Hanel M, Kobbe G, et al. AntiCD20 immunotherapy as a bridge to tolerance, after allogeneic stem cell transplantation for patients with chronic lymphocytic leukaemia: results of the CLLX4 trial. $\mathrm{Br} \mathrm{J}$ Haematol 2019; 184:833-6.

2. Qi J, Li X, Peng H, Cook EM, Dadashian EL, Wiestner A, et al. Potent and selective antitumor activity of a $\mathrm{T}$ cell-engaging bispecific antibody targeting a membrane-proximal epitope of ROR1. Proc Natl Acad Sci U S A 2018; 115:E5467-E76.

3. Dreier T, Baeuerle PA, Fichtner I, Grun M, Schlereth B, Lorenczewski G, et al. T cell costimulus-independent and very efficacious inhibition of tumor growth in mice bearing subcutaneous or leukemic human B cell lymphoma xenografts by a CD19-/CD3bispecific single-chain antibody construct. J Immunol 2003; 170:4397-402.

4. Sun LL, Ellerman D, Mathieu M, Hristopoulos M, Chen X, Li Y, et al. AntiCD20/CD3 T cell-dependent bispecific antibody for the treatment of B cell malignancies. Sci Trans1 Med 2015; 7:287ra70.

5. Lee NK, Zhang Y, Su Y, Bidlingmaier S, Sherbenou DW, Ha KD, et al. Cell-type specific potent Wnt signaling blockade by bispecific antibody. Scientific reports 2018; $8: 766$.

6. Lee NK, Su Y, Bidlingmaier S, Liu B. Manipulation of cell-type selective antibody internalization by a guide-effector bispecific design. Molecular cancer therapeutics 2019. 
7. de Goeij BE, Vink T, Ten Napel H, Breij EC, Satijn D, Wubbolts R, et al. Efficient Payload Delivery by a Bispecific Antibody-Drug Conjugate Targeting HER2 and CD63. Molecular cancer therapeutics 2016; 15:2688-97.

8. Laursen NS, Friesen RHE, Zhu X, Jongeneelen M, Blokland S, Vermond J, et al. Universal protection against influenza infection by a multidomain antibody to influenza hemagglutinin. Science 2018; 362:598-602.

9. Huang Y, Yu J, Lanzi A, Yao X, Andrews CD, Tsai L, et al. Engineered Bispecific Antibodies with Exquisite HIV-1-Neutralizing Activity. Cell 2016; 165:162131.

10. Wagner EK, Wang X, Bui A, Maynard JA. Synergistic Neutralization of Pertussis Toxin by a Bispecific Antibody In Vitro and In Vivo. Clinical and vaccine immunology : CVI 2016; 23:851-62.

11. Kontermann RE. Dual targeting strategies with bispecific antibodies. MAbs 2012; 4:182-97.

12. Kipriyanov SM, Cochlovius B, Schafer HJ, Moldenhauer G, Bahre A, Le Gall F, et al. Synergistic antitumor effect of bispecific CD19 x CD3 and CD19 x CD16 diabodies in a preclinical model of non-Hodgkin's lymphoma. J Immunol 2002; 169:137-44.

13. Qu Z, Goldenberg DM, Cardillo TM, Shi V, Hansen HJ, Chang CH. Bispecific anti-CD20/22 antibodies inhibit B-cell lymphoma proliferation by a unique mechanism of action. Blood 2008; 111:2211-9.

14. Friedman M, Lindstrom S, Ekerljung L, Andersson-Svahn H, Carlsson J, Brismar $\mathrm{H}$, et al. Engineering and characterization of a bispecific HER2 x EGFR-binding affibody molecule. Biotechnol Appl Biochem 2009; 54:121-31. 
15. Wu C, Ying H, Bose S, Miller R, Medina L, Santora L, et al. Molecular construction and optimization of anti-human IL-1alpha/beta dual variable domain immunoglobulin (DVD-Ig) molecules. mAbs 2009; 1:339-47.

16. Wu C, Ying H, Grinnell C, Bryant S, Miller R, Clabbers A, et al. Simultaneous targeting of multiple disease mediators by a dual-variable-domain immunoglobulin. Nature biotechnology 2007; 25:1290-7.

17. Schaefer W, Regula JT, Bahner M, Schanzer J, Croasdale R, Durr H, et al. Immunoglobulin domain crossover as a generic approach for the production of bispecific IgG antibodies. Proc Natl Acad Sci U S A 2011; 108:11187-92.

18. Sedykh SE, Prinz VV, Buneva VN, Nevinsky GA. Bispecific antibodies: design, therapy, perspectives. Drug design, development and therapy 2018; 12:195-208.

19. Krishnamurthy A, Jimeno A. Bispecific antibodies for cancer therapy: A review. Pharmacology \& therapeutics 2018; 185:122-34.

20. Montefiori DC. Bispecific Antibodies Against HIV. Cell 2016; 165:1563-4.

21. Kontermann RE. Strategies for extended serum half-life of protein therapeutics. Current opinion in biotechnology 2011; 22:868-76.

22. Niewoehner J, Bohrmann B, Collin L, Urich E, Sade H, Maier P, et al. Increased brain penetration and potency of a therapeutic antibody using a monovalent molecular shuttle. Neuron 2014; 81:49-60.

23. Brinkmann U, Kontermann RE. The making of bispecific antibodies. mAbs 2017; 9:182-212.

24. Thakur A, Lum LG. "NextGen" Biologics: Bispecific Antibodies and Emerging Clinical Results. Expert opinion on biological therapy 2016; 16:675-88. 
25. Brennan M, Davison PF, Paulus H. Preparation of bispecific antibodies by chemical recombination of monoclonal immunoglobulin G1 fragments. Science 1985; 229:81-3.

26. Staerz UD, Kanagawa O, Bevan MJ. Hybrid antibodies can target sites for attack by T cells. Nature $1985 ; 314: 628-31$.

27. Hayden MS, Linsley PS, Gayle MA, Bajorath J, Brady WA, Norris NA, et al. Single-chain mono- and bispecific antibody derivatives with novel biological properties and antitumour activity from a COS cell transient expression system. Therapeutic immunology 1994; 1:3-15.

28. Mallender WD, Voss EW, Jr. Construction, expression, and activity of a bivalent bispecific single-chain antibody. The Journal of biological chemistry 1994; 269:199-206. 29. Holliger P, Prospero T, Winter G. "Diabodies": small bivalent and bispecific antibody fragments. Proceedings of the National Academy of Sciences of the United States of America 1993; 90:6444-8.

30. Schoonjans R, Willems A, Schoonooghe S, Fiers W, Grooten J, Mertens N. Fab chains as an efficient heterodimerization scaffold for the production of recombinant bispecific and trispecific antibody derivatives. Journal of immunology 2000; 165:7050-7.

31. Schoonjans R, Willems A, Schoonooghe S, Leoen J, Grooten J, Mertens N. A new model for intermediate molecular weight recombinant bispecific and trispecific antibodies by efficient heterodimerization of single chain variable domains through fusion to a Fab-chain. Biomolecular engineering 2001; 17:193-202. 
32. Lu D, Jimenez X, Zhang H, Bohlen P, Witte L, Zhu Z. Fab-scFv fusion protein: an efficient approach to production of bispecific antibody fragments. Journal of immunological methods 2002; 267:213-26.

33. Chang $\mathrm{CH}$, Rossi EA, Goldenberg DM. The dock and lock method: a novel platform technology for building multivalent, multifunctional structures of defined composition with retained bioactivity. Clinical cancer research : an official journal of the American Association for Cancer Research 2007; 13:5586s-91s.

34. Klein C, Sustmann C, Thomas M, Stubenrauch K, Croasdale R, Schanzer J, et al. Progress in overcoming the chain association issue in bispecific heterodimeric $\operatorname{IgG}$ antibodies. mAbs 2012; 4:653-63.

35. Castoldi R, Schanzer J, Panke C, Jucknischke U, Neubert NJ, Croasdale R, et al. TetraMabs: simultaneous targeting of four oncogenic receptor tyrosine kinases for tumor growth inhibition in heterogeneous tumor cell populations. Protein engineering, design \& selection : PEDS 2016; 29:467-75.

36. Schmidt DJ, Beamer G, Tremblay JM, Steele JA, Kim HB, Wang Y, et al. A Tetraspecific VHH-Based Neutralizing Antibody Modifies Disease Outcome in Three Animal Models of Clostridium difficile Infection. Clinical and vaccine immunology : CVI 2016; 23:774-84.

37. Hu S, Fu W, Xu W, Yang Y, Cruz M, Berezov SD, et al. Four-in-one antibodies have superior cancer inhibitory activity against EGFR, HER2, HER3, and VEGF through disruption of HER/MET crosstalk. Cancer research 2015; 75:159-70.

38. Klinger M, Brandl C, Zugmaier G, Hijazi Y, Bargou RC, Topp MS, et al. Immunopharmacologic response of patients with B-lineage acute lymphoblastic leukemia 
to continuous infusion of $\mathrm{T}$ cell-engaging CD19/CD3-bispecific BiTE antibody blinatumomab. Blood 2012; 119:6226-33.

39. Jain T, Sun T, Durand S, Hall A, Houston NR, Nett JH, et al. Biophysical properties of the clinical-stage antibody landscape. Proc Natl Acad Sci U S A 2017; 114:944-9.

40. Orcutt KD, Ackerman ME, Cieslewicz M, Quiroz E, Slusarczyk AL, Frangioni JV, et al. A modular IgG-scFv bispecific antibody topology. Protein engineering, design \& selection : PEDS 2010; 23:221-8.

41. Ridgway JB, Presta LG, Carter P. 'Knobs-into-holes' engineering of antibody CH3 domains for heavy chain heterodimerization. Protein engineering 1996; 9:617-21.

42. Atwell S, Ridgway JB, Wells JA, Carter P. Stable heterodimers from remodeling the domain interface of a homodimer using a phage display library. Journal of molecular biology 1997; 270:26-35.

43. Dall'Acqua W, Simon AL, Mulkerrin MG, Carter P. Contribution of domain interface residues to the stability of antibody $\mathrm{CH} 3$ domain homodimers. Biochemistry 1998; 37:9266-73.

44. Yin Y, Han G, Zhou J, Dillon M, McCarty L, Gavino L, et al. Precise quantification of mixtures of bispecific $\operatorname{IgG}$ produced in single host cells by liquid chromatography-Orbitrap high-resolution mass spectrometry. mAbs 2016; 8:1467-76.

45. Von Kreudenstein TS, Escobar-Carbrera E, Lario PI, D'Angelo I, Brault K, Kelly $\mathrm{J}$, et al. Improving biophysical properties of a bispecific antibody scaffold to aid developability: quality by molecular design. mAbs 2013; 5:646-54. 
46. Merchant AM, Zhu Z, Yuan JQ, Goddard A, Adams CW, Presta LG, et al. An efficient route to human bispecific IgG. Nature biotechnology 1998; 16:677-81.

47. Elliott JM, Ultsch M, Lee J, Tong R, Takeda K, Spiess C, et al. Antiparallel conformation of knob and hole aglycosylated half-antibody homodimers is mediated by a CH2-CH3 hydrophobic interaction. Journal of molecular biology 2014; 426:1947-57.

48. Wei H, Cai H, Jin Y, Wang P, Zhang Q, Lin Y, et al. Structural basis of a novel heterodimeric Fc for bispecific antibody production. Oncotarget 2017; 8:51037-49.

49. Zhu Z, Presta LG, Zapata G, Carter P. Remodeling domain interfaces to enhance heterodimer formation. Protein science : a publication of the Protein Society 1997; 6:7818.

50. Carter P. Bispecific human IgG by design. Journal of immunological methods $2001 ; 248: 7-15$.

51. Van Blarcom T, Lindquist K, Melton Z, Cheung WL, Wagstrom C, McDonough D, et al. Productive common light chain libraries yield diverse panels of high affinity bispecific antibodies. mAbs 2018; 10:256-68.

52. Krah S, Schroter C, Eller C, Rhiel L, Rasche N, Beck J, et al. Generation of human bispecific common light chain antibodies by combining animal immunization and yeast display. Protein engineering, design \& selection : PEDS 2017; 30:291-301.

53. Klein C, Schaefer W, Regula JT. The use of CrossMAb technology for the generation of bi- and multispecific antibodies. mAbs 2016; 8:1010-20.

54. Steinwand M, Droste P, Frenzel A, Hust M, Dubel S, Schirrmann T. The influence of antibody fragment format on phage display based affinity maturation of IgG. mAbs 2014; 6:204-18. 
55. Schirrmann T, Menzel C, Hust M, Prilop J, Jostock T, Dubel S. Oligomeric forms of single chain immunoglobulin (scIgG). mAbs 2010; 2:73-6.

56. Koerber JT, Hornsby MJ, Wells JA. An improved single-chain Fab platform for efficient display and recombinant expression. Journal of molecular biology 2015; 427:576-86.

57. Wranik BJ, Christensen EL, Schaefer G, Jackman JK, Vendel AC, Eaton D. LUZY, a novel platform for the mammalian cell production of full-length IgG-bispecific antibodies. The Journal of biological chemistry 2012; 287:43331-9.

58. Dimasi N, Fleming R, Sachsenmeier KF, Bezabeh B, Hay C, Wu J, et al. Guiding bispecific monovalent antibody formation through proteolysis of IgG1 single-chain. mAbs 2017; 9:438-54.

59. Funaro A, Reinis M, Trubiani O, Santi S, Di Primio R, Malavasi F. CD38 functions are regulated through an internalization step. Journal of immunology 1998; 160:2238-47.

60. Yamamoto K, Ichijo H, Korsmeyer SJ. BCL-2 is phosphorylated and inactivated by an ASK1/Jun N-terminal protein kinase pathway normally activated at G(2)/M. Mol Cell Biol 1999; 19:8469-78.

61. Contardi E, Palmisano GL, Tazzari PL, Martelli AM, Fala F, Fabbi M, et al. CTLA-4 is constitutively expressed on tumor cells and can trigger apoptosis upon ligand interaction. International journal of cancer 2005; 117:538-50.

62. Smith K, Garman L, Wrammert J, Zheng NY, Capra JD, Ahmed R, et al. Rapid generation of fully human monoclonal antibodies specific to a vaccinating antigen. Nat Protoc $2009 ; 4: 372-84$. 
bioRxiv preprint doi: https://doi.org/10.1101/2019.12.25.888586; this version posted December 27, 2019. The copyright holder for this preprint (which was not certified by peer review) is the author/funder, who has granted bioRxiv a license to display the preprint in perpetuity. It is made available under aCC-BY-NC-ND 4.0 International license.

63. Li C, Evans RM. Ligation independent cloning irrespective of restriction site compatibility. Nucleic acids research 1997; 25:4165-6. 


\section{Figure legends}

Fig. 1 Monoclonal antibodies processed from single-chain IgGs show similar biophysical and binding properties as the original antibodies. (A) The scheme to produce monoclonal IgG from single-chain IgG with a thrombin cleavable linker. (B) After thrombin cleavage, the IgG molecules produced with the thrombin-cleavable linker (Ipili-sc36TMB and Dara-sc36TMB) show molecular weights of approximately $150 \mathrm{kDa}$ and can be separated into heavy chains $(\sim 50 \mathrm{kDa})$ and light chains $(\sim 25 \mathrm{kDa})$ with a reducing agent, $\beta$-mercaptoethanol ( $\beta$-ME), a virtually identical pattern compared with the original monoclonal antibody. MW: approximate molecular weight marker. (C) Ipilisc36TMB and (D) Dara-sc36TMB bind to their respective ligands in ELISA assays, showing similar binding profiles compared with the original monoclonal antibodies.

\section{Fig. 2 Bispecific antibodies produced with thrombin-cleavable linkers and KIH Fc}

domains. (A) The scheme to produce bispecific antibodies. (B) Two bispecific antibodies, Ipili-Dara-KIH and Ipili-Her-KIH, were generated, both of which show a predominant component with a molecular weight of $150 \mathrm{kDa}$. A small fraction of contamination, likely caused by incorrectly paired homodimers, can be observed at the half size of an antibody (labeled with '*'). Under reducing condition, separated heavy and light chains, $\sim 50 \mathrm{kDa}$ and $\sim 25 \mathrm{kDa}$ respectively, were observed. (C, D) Bispecific Ipili-Dara-KIH binding to CTLA4-Fc (C) and CD38 (D), in comparison to the original monoclonal antibodies. (E, F) Bispecific Ipili-Her-KIH binding to CTLA4-Fc (E) and ErbB2-Fc (F), in comparison to the original monoclonal antibodies. 


\section{Fig. 3 Bispecific antibody with high purity produced with a pair of thrombin-} cleavable dual-tagged affinity linkers. (A) The scheme to produce bispecific antibodies with built-in affinity tags, e.g., 10-His tag and Twin-Strep-Tag ${ }^{\circledR}$ that enables sequential purification of the desired heterodimer by Ni-NTA resin and Strep-Tactin ${ }^{\circledR}$ XT resin. (B) The bispecific product of Ipili-Dara-KIH purified via the dual tags (Ni-NTA and Strep$\operatorname{Tactin}^{\circledR} \mathrm{XT}$ ) is compared with the same product purified by protein A agarose. The contamination in the bispecific products that can be observed at the half-antibody size (the '*' band) is eliminated when purified with dual tags. (C, D) The purity of bispecific products is assessed by analytical HIC. (E) Flow cytometry analysis of Jurkat cell binding by Ipili-Dara-KIH, Daratumumab, and Ipilimumab at various concentrations (0 $200 \mathrm{nM}$ ). (F) Flow cytometry analysis of MCF7 cell binding by Ipili-Her-KIH, Herceptin, and Ipilimumab at various concentrations (0 - $200 \mathrm{nM})$.

Fig. 4 Design of Tri-N-Fabs as a new format for tri-specific antibodies produced with thrombin-removable linkers and KIH Fc heterodimers. The production scheme and final structure of the Tri-N-Fabs is presented in (A). The extra Fab domain (III) is appended to the N-terminus of the bispecific antibody. For the Tri-N-Fabs produced, we

placed Fabs of Ipilimumab at position I, Daratumumab at position II, and Herceptin at position III. (B) On SDS-PAGE, the Tri-N-Fabs shows an approximate molecular weight of $200 \mathrm{kDa}$ and can be separated into polypeptide chains of $\sim 50 \mathrm{kDa}$ and $\sim 25 \mathrm{kDa}$ under reducing conditions. Small fractions of contamination were observed, marked as $(*)$ and 
$(\dagger)$, likely caused by homodimers. (C) The purity of the Tri-N-Fabs is assessed by analytical hydrophobic interaction chromatography. (D, E, F) Tri-N-Fabs interacts with the three intended ligands in an ELISA assay. Parental antibodies were included as references.

Fig. 5 Tetra-N-Fabs, a new format for tetra-specific antibodies, is produced with thrombin-removable linkers and KIH Fc heterodimers. (A) The design of Tetra-NFabs. Extra Fab domains (III and IV) are appended to the N-terminus of the bispecific antibody. For the Tetra-N-Fabs produced, we placed Fabs of Ipilimumab at position I, Daratumumab at position II, Herceptin at position III, and Atezolizumab at position IV. (B) The Tetra-N-Fabs displays a molecular weight of $\sim 250 \mathrm{kDa}$ by non-reducing SDSPAGE analysis and can be separated into two polypeptide chains of $\sim 50 \mathrm{kDa}$ and $\sim 25$ $\mathrm{kDa}$ under reducing conditions. (C) The purity of the Tetra-N-Fabs is estimated to be 95\% by analytical HIC. (D, E, F, G) Binding of the Tetra-N-Fabs to all four ligands with parental antibodies included as references.

Fig. 6 Tri-C-Fabs and Tetra-C-Fabs are alternative formats for tri- and tetraspecific antibody generation using the thrombin-removable linker and KIH Fc heterodimer. Schematic presentations of Tri-C-Fabs (A) and Tetra-C-Fabs (B). Fab domains representing the $3^{\text {rd }}$ and $4^{\text {th }}$ specificity were appended to the C-terminus of the bispecific antibody. We placed Fabs of Ipilimumab at position I, Daratumumab at position II, Herceptin at position III, and Atezolizumab at position IV (in the case of Tetra-C-Fabs). (C) SDS-PAGE analysis of Tri-C-Fabs and Tetra-C-Fabs. The Tri-C-Fabs 
displays an approximate molecular weight of $200 \mathrm{kDa}$ and can be separated into polypeptide chains of $\sim 75 \mathrm{kDa}, \sim 50 \mathrm{kDa}$, and $\sim 25 \mathrm{kDa}$ under reducing conditions. The Tetra-C-Fabs shows an approximate molecular weight of $250 \mathrm{kDa}$ and can be separated into polypeptide chains of $\sim 75 \mathrm{kDa}$ and $\sim 25 \mathrm{kDa}$ under reducing conditions. (D, E, F) The Tri-C-Fabs binds to all three intended ligands by ELISA, with the parental antibodies included as references. (G, H, I, J) Binding of the Tetra-C-Fabs to all four intended ligands by ELISA, with parental antibodies included as references. 
bioRxiv preprint doi: https://doi.org/10.1101/2019.12 25.888586; this version posted December 27, 2019. The copyright holder for this preprint (which was not certified by peer review) is the author/funder, who has granted bioRxiv a license to display the preprint in perpetuity. It is made available under aCC-BY-NC-ND 4.0 International license.

Figure 1

A

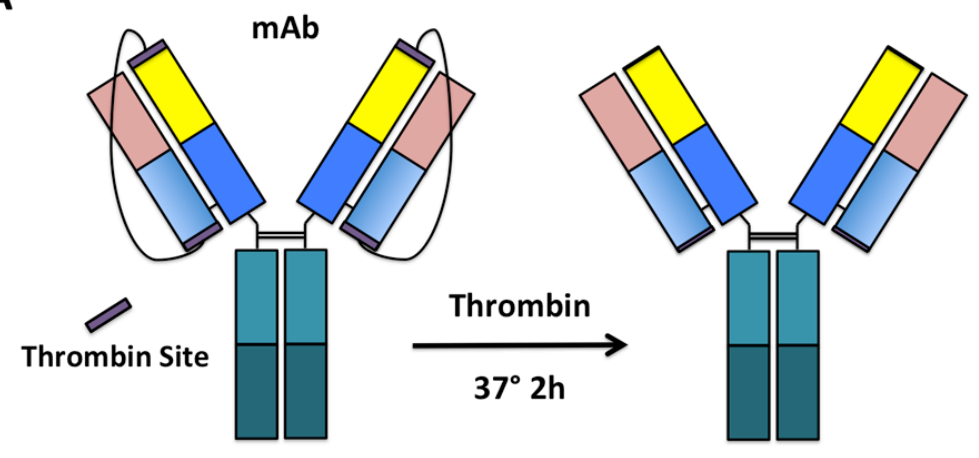

C

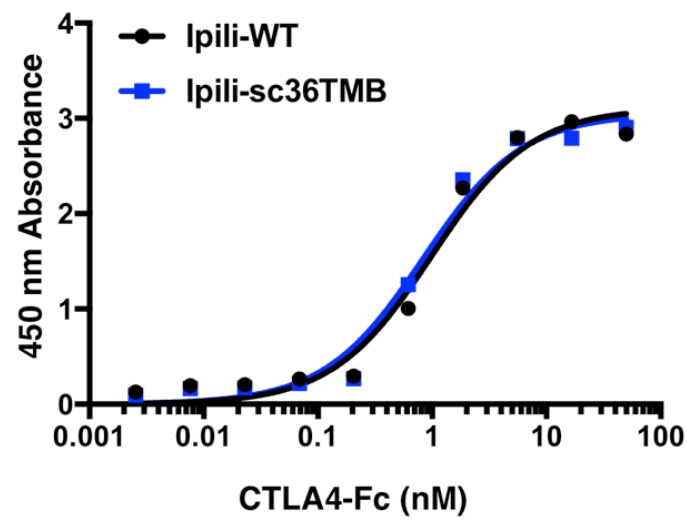

B

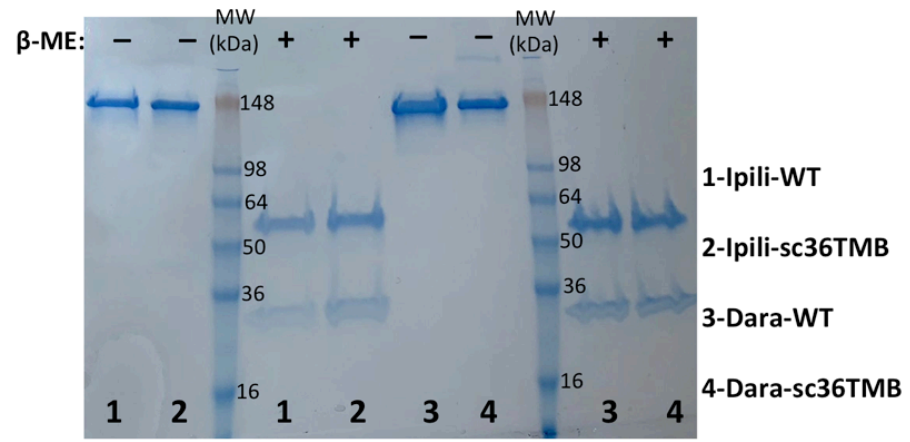

D

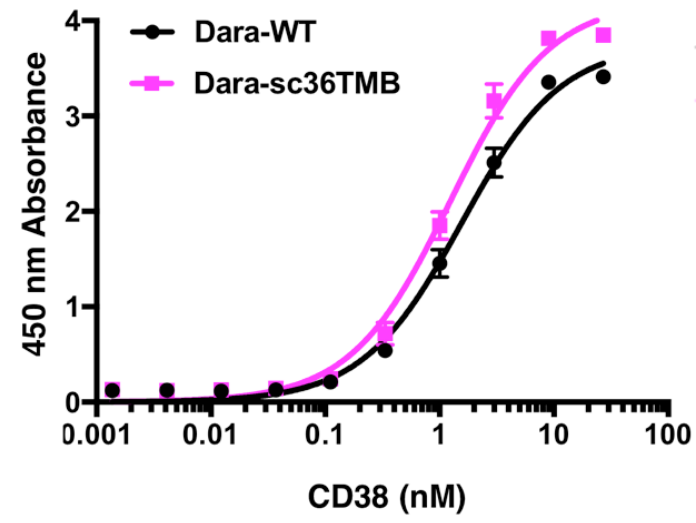




\section{Figure 2}

A

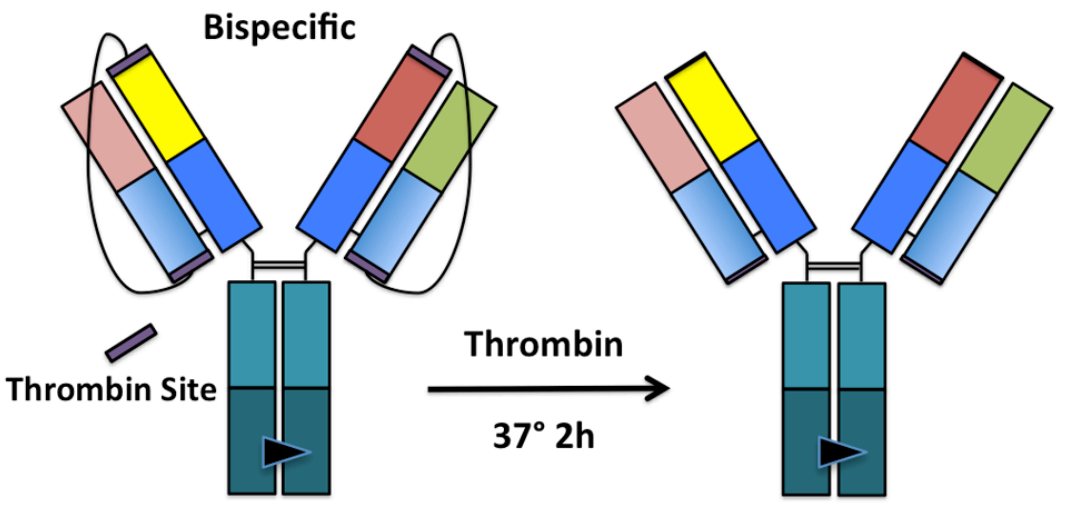

B

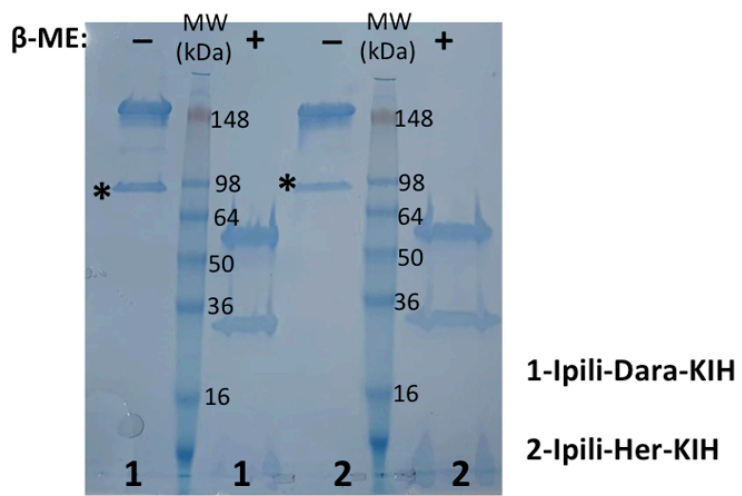

C

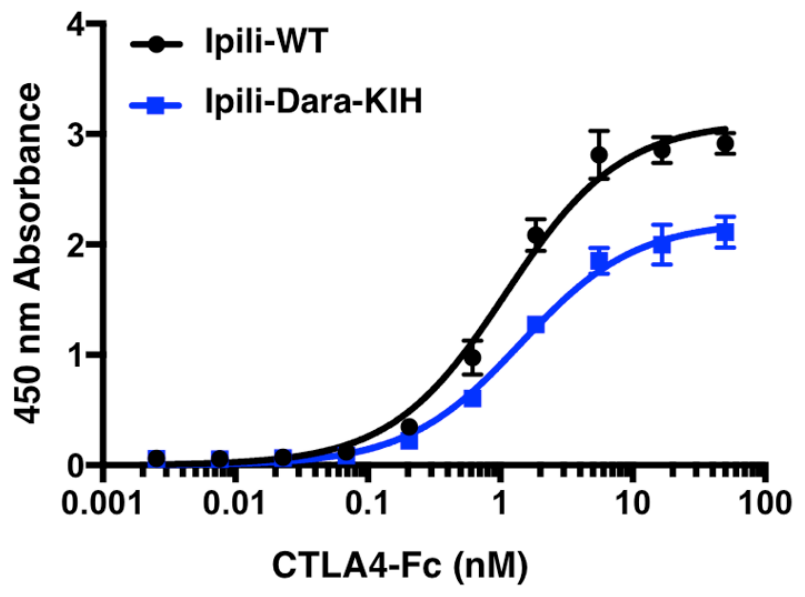

$\mathbf{E}$

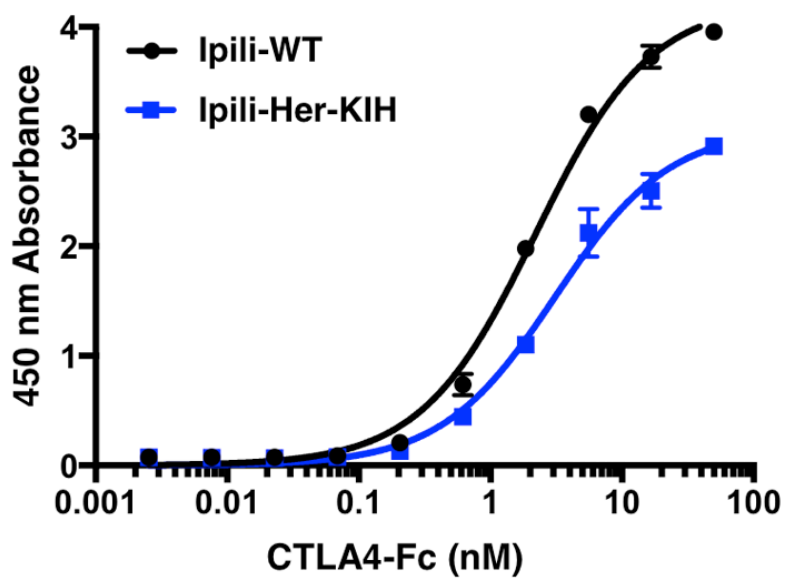

D

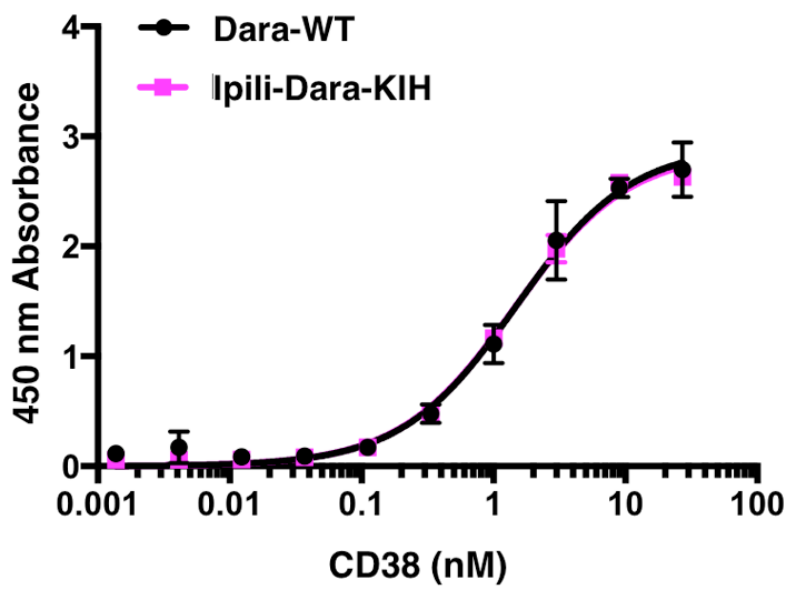

F

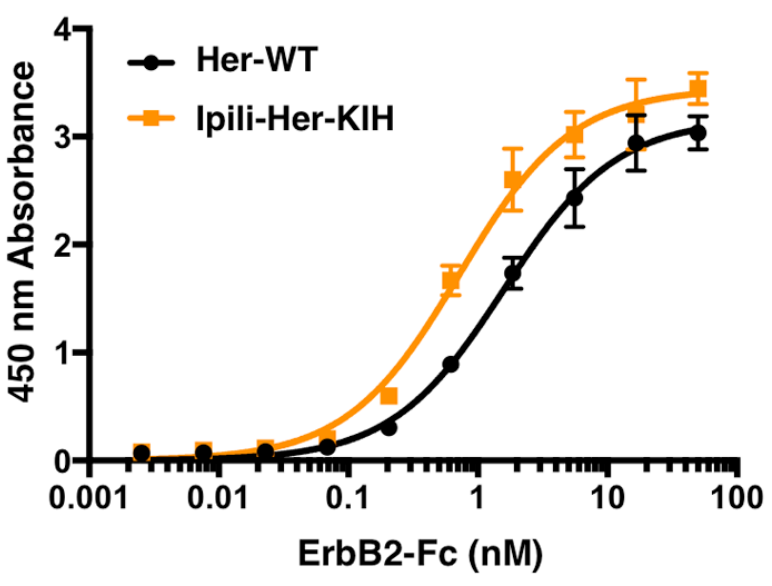


A

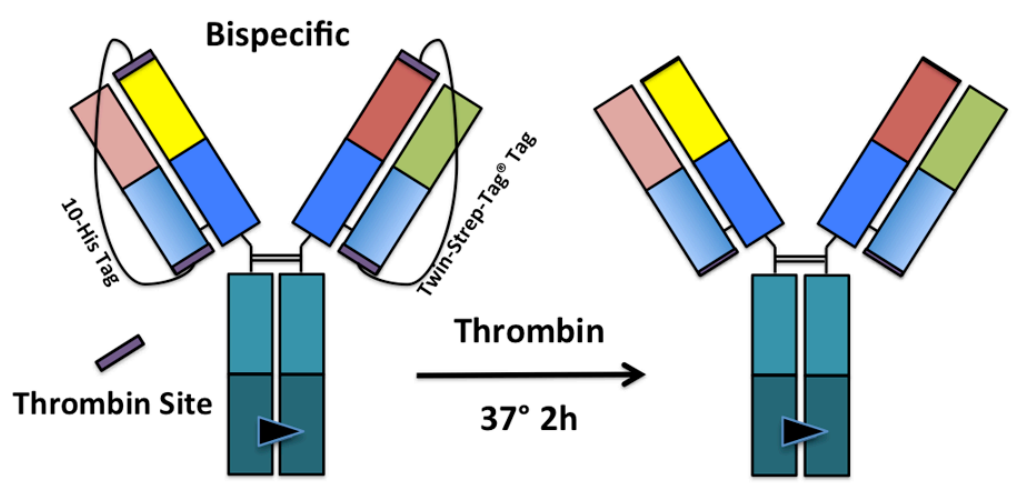

C

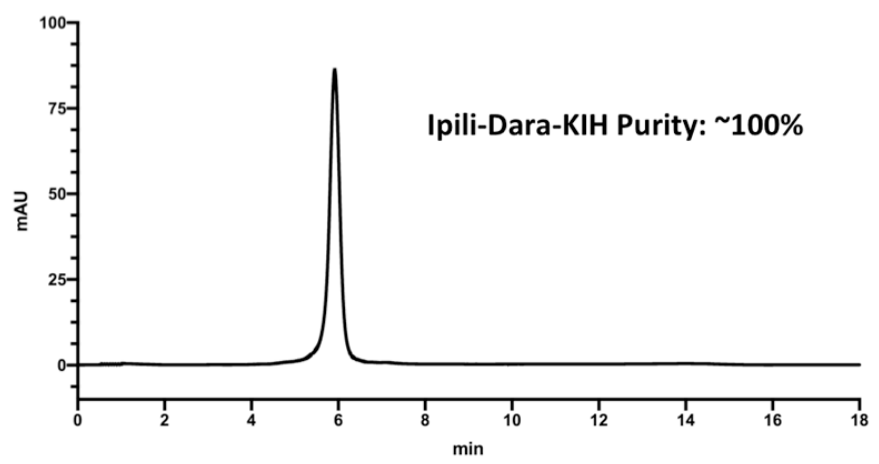

$\mathbf{E}$

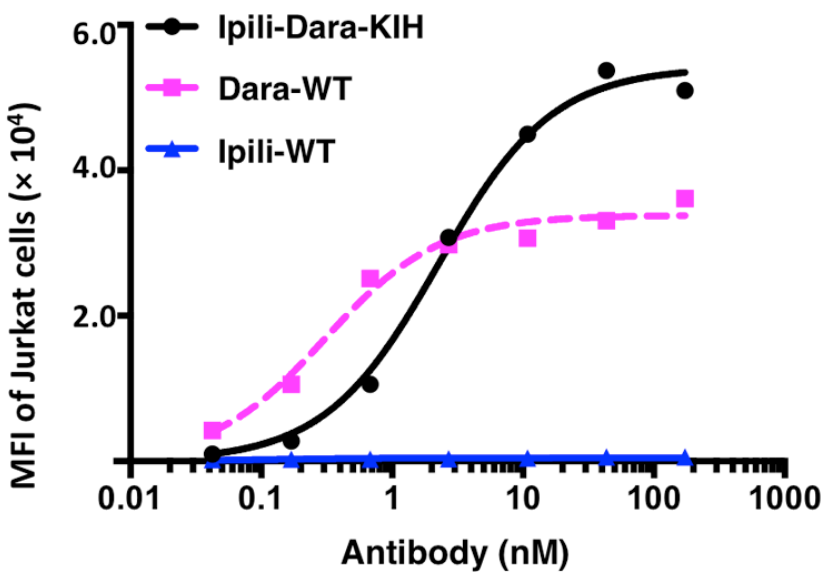

B

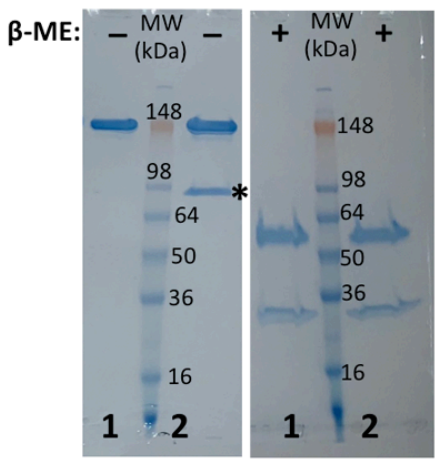

1-Ipili-Dara-KIH (Dual tags-purified)

2-Ipli-Dara-KIH (Total IgG)

D

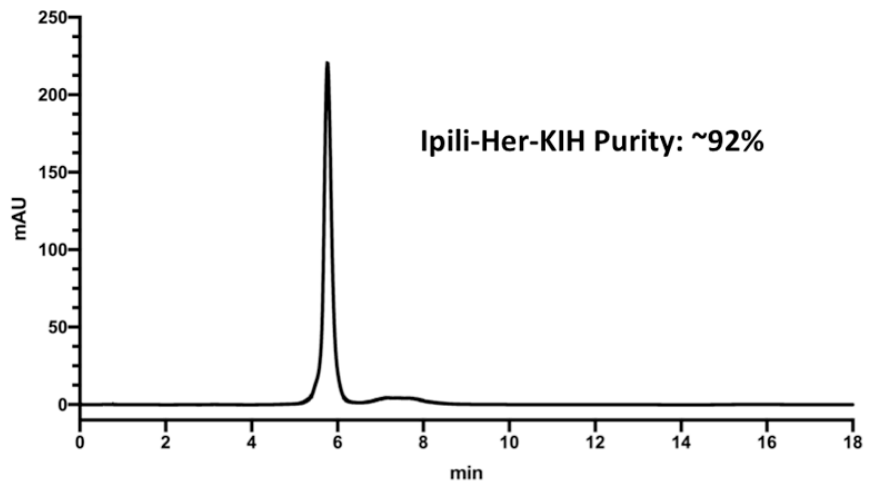

F

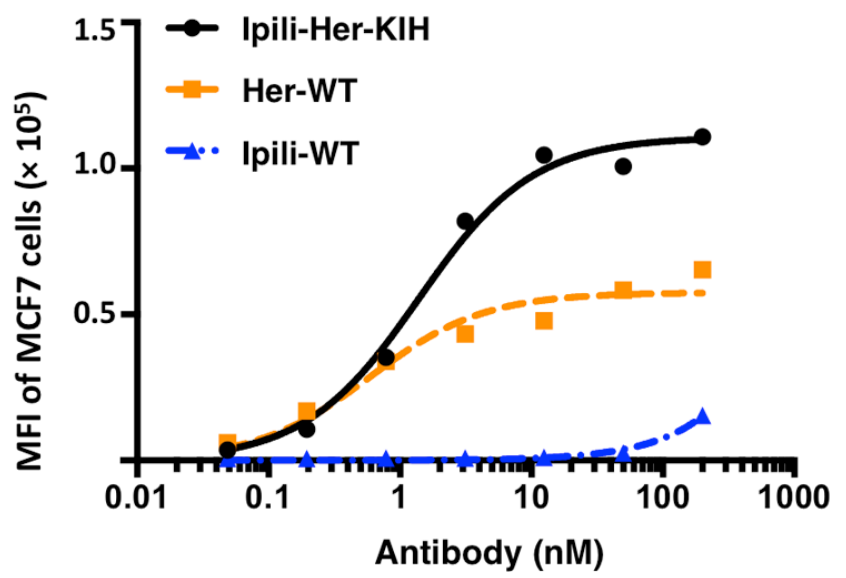


bioRxiv preprint doi: https://doi.org/10.1101/2019.12.25.888586; this version posted December 27, 2019. The copyright holder for this preprint (which was not certified by peer review) is the author/funder, who has granted bioRxiv a license to display the preprint in perpetuity. It is made available under aCC-BY-NC-ND 4.0 International license.

Figure 4

A

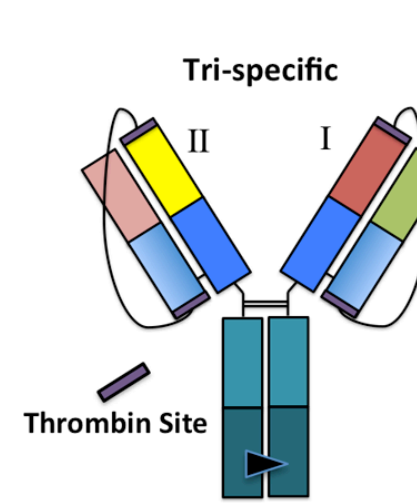

B

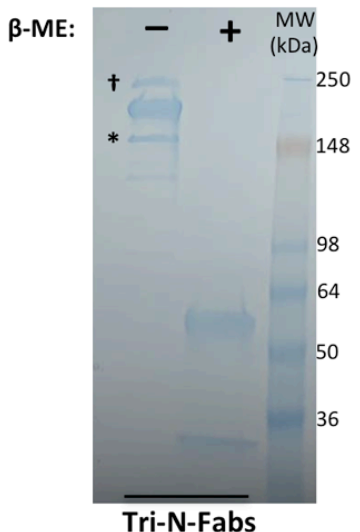

D

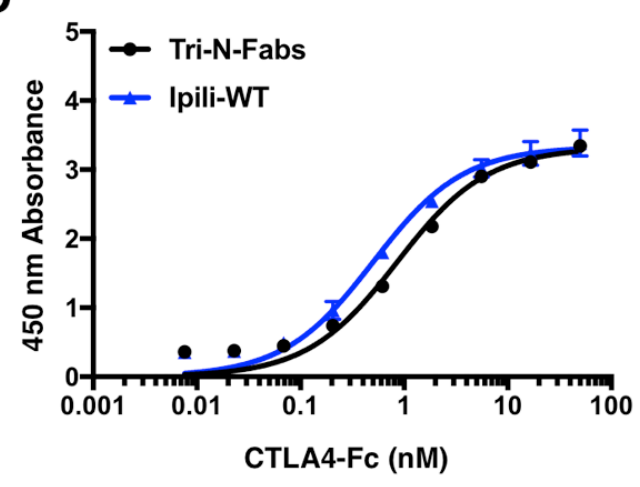

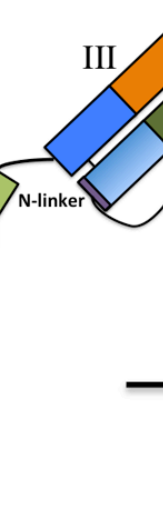

C

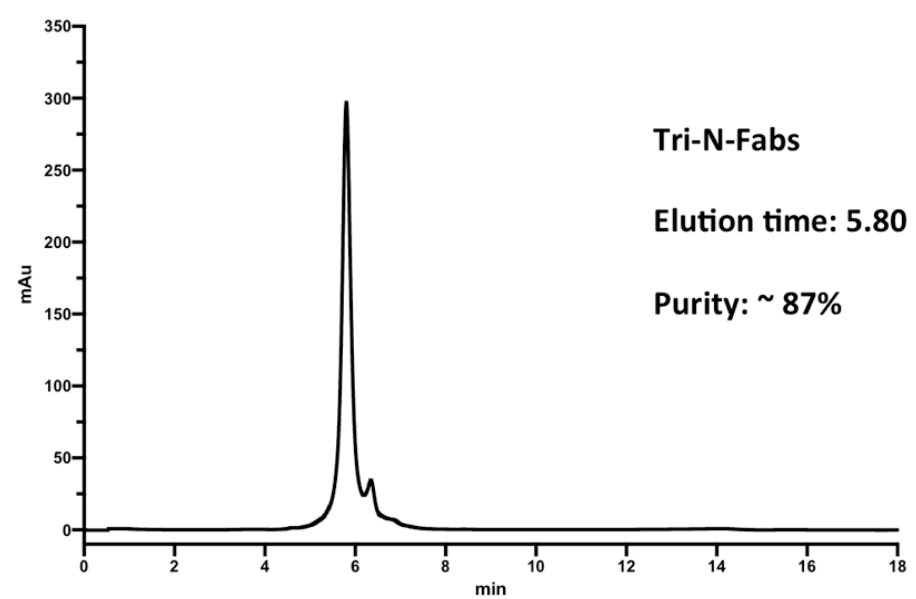

$\mathbf{E}$

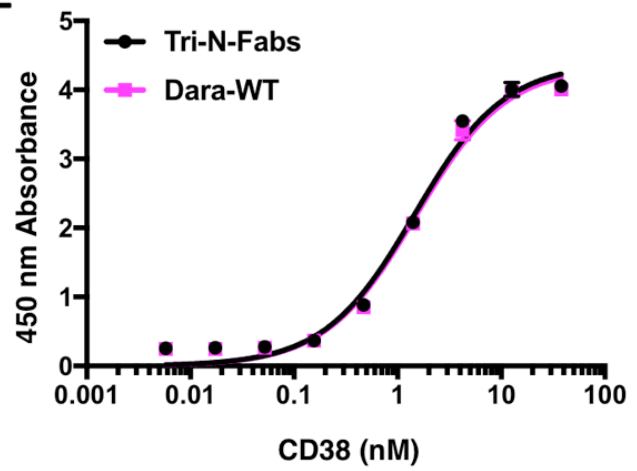

$\mathbf{F}$

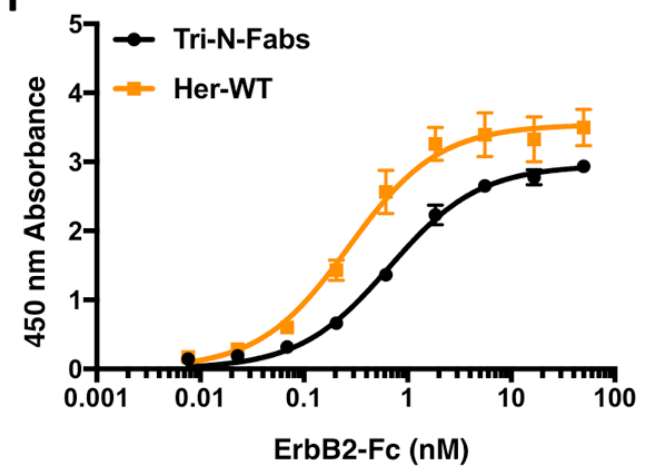


A

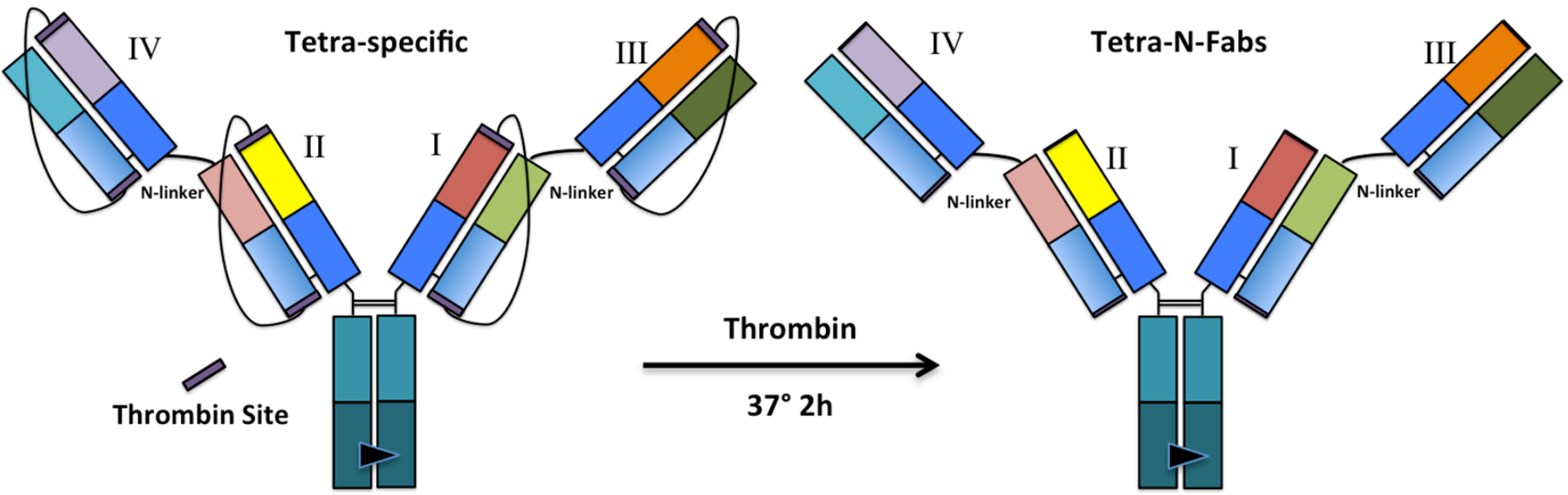

B

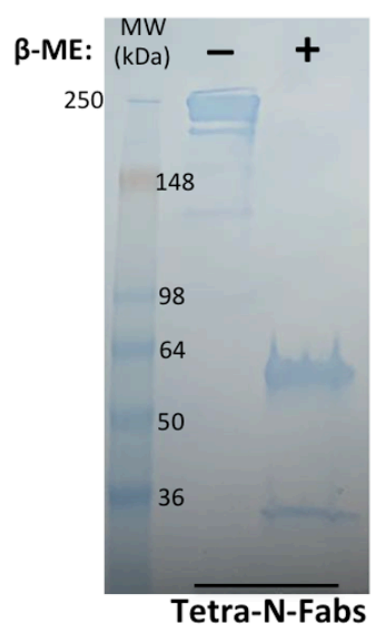

C

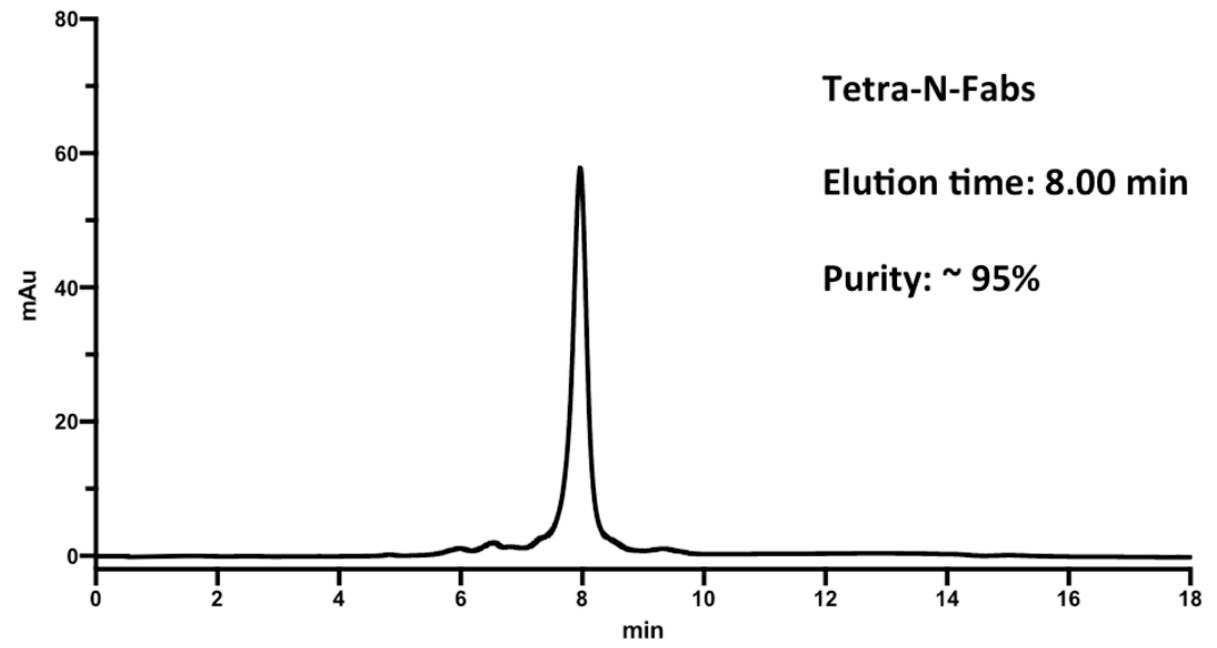

D

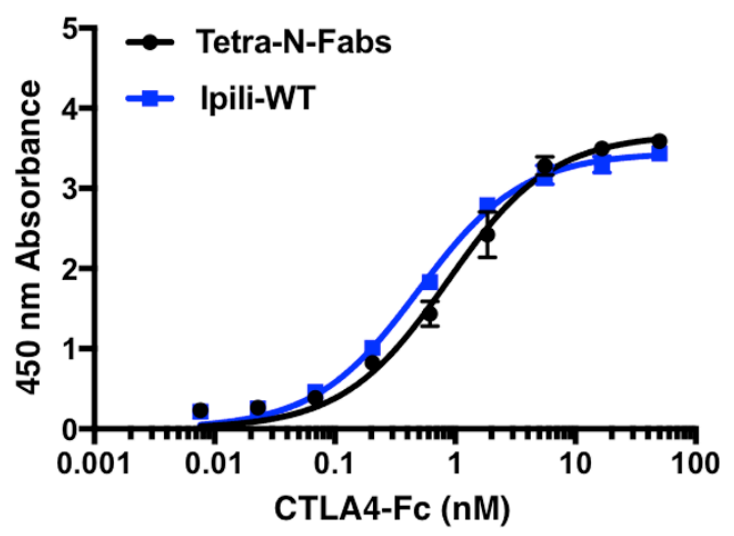

F

E
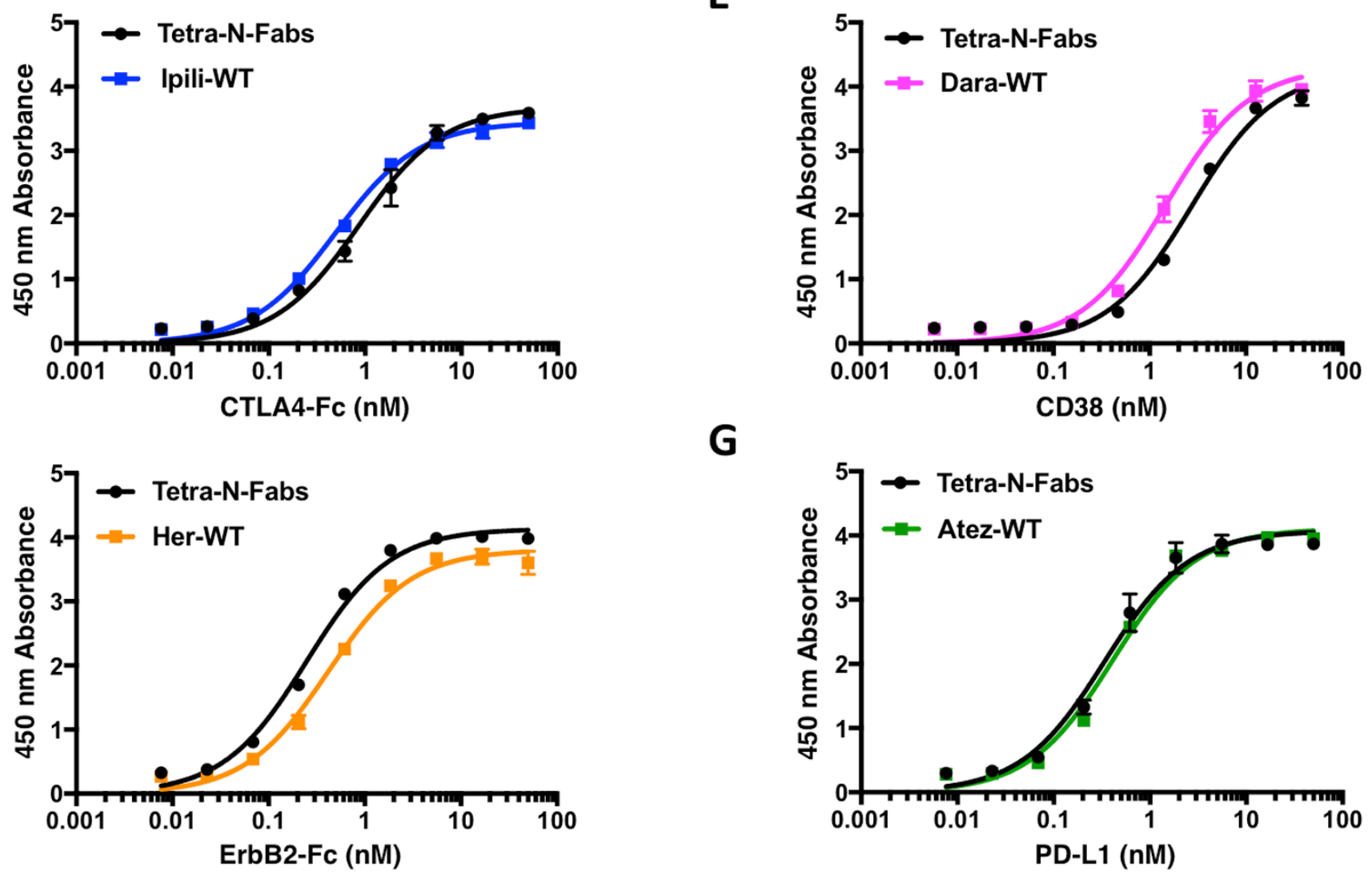

G

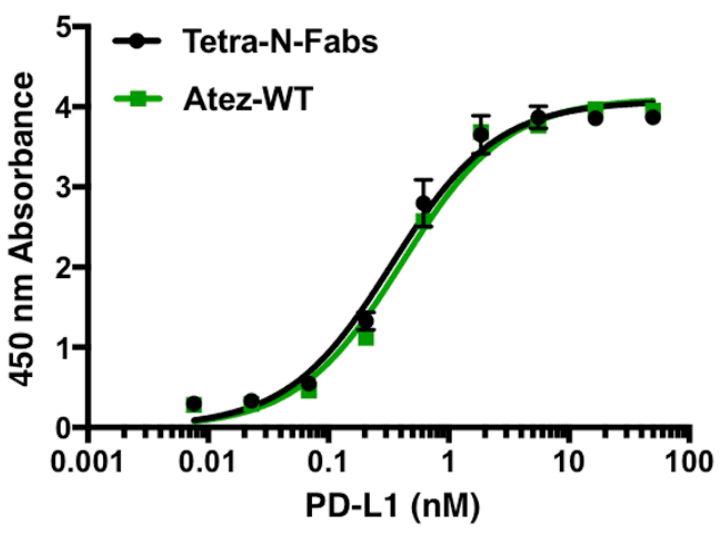


bioRxiv preprint doi: https://doi.org/10.1101/2019.12.25 888586; this version posted December 27, 2019. The copyright holder for this preprint (which was not certified by peer review) is the author/funder, who has granted bioRxiv a license to display the preprint in perpetuity. It is made available under aCC-BY-NC-ND 4.0 International license.

\section{Figure 6}

A

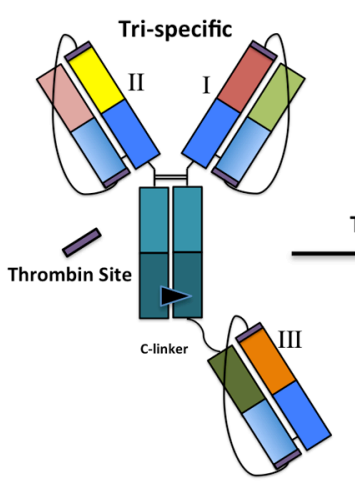

C

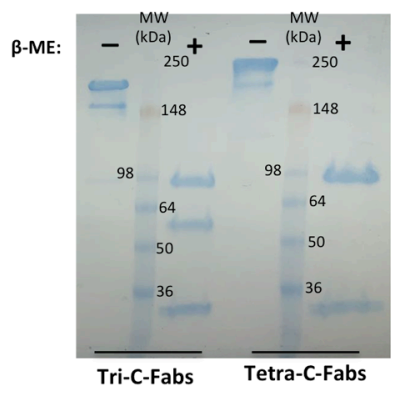

G

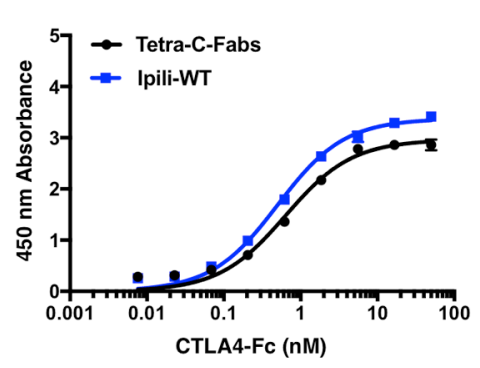

ri-C-Fabs

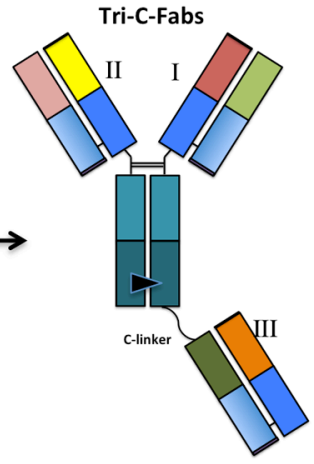

B

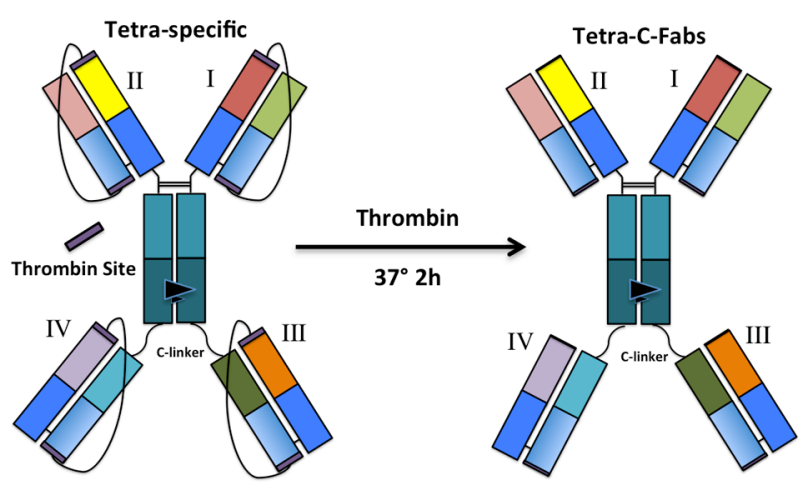

D

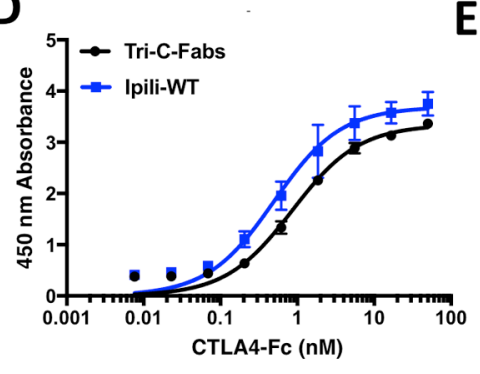

E

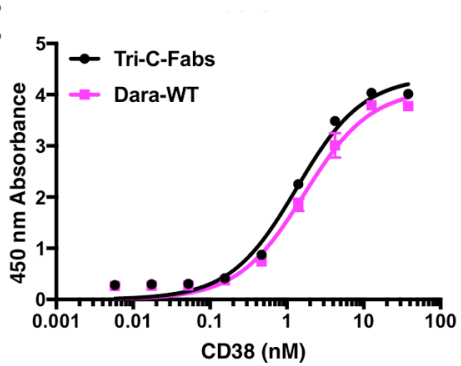

F
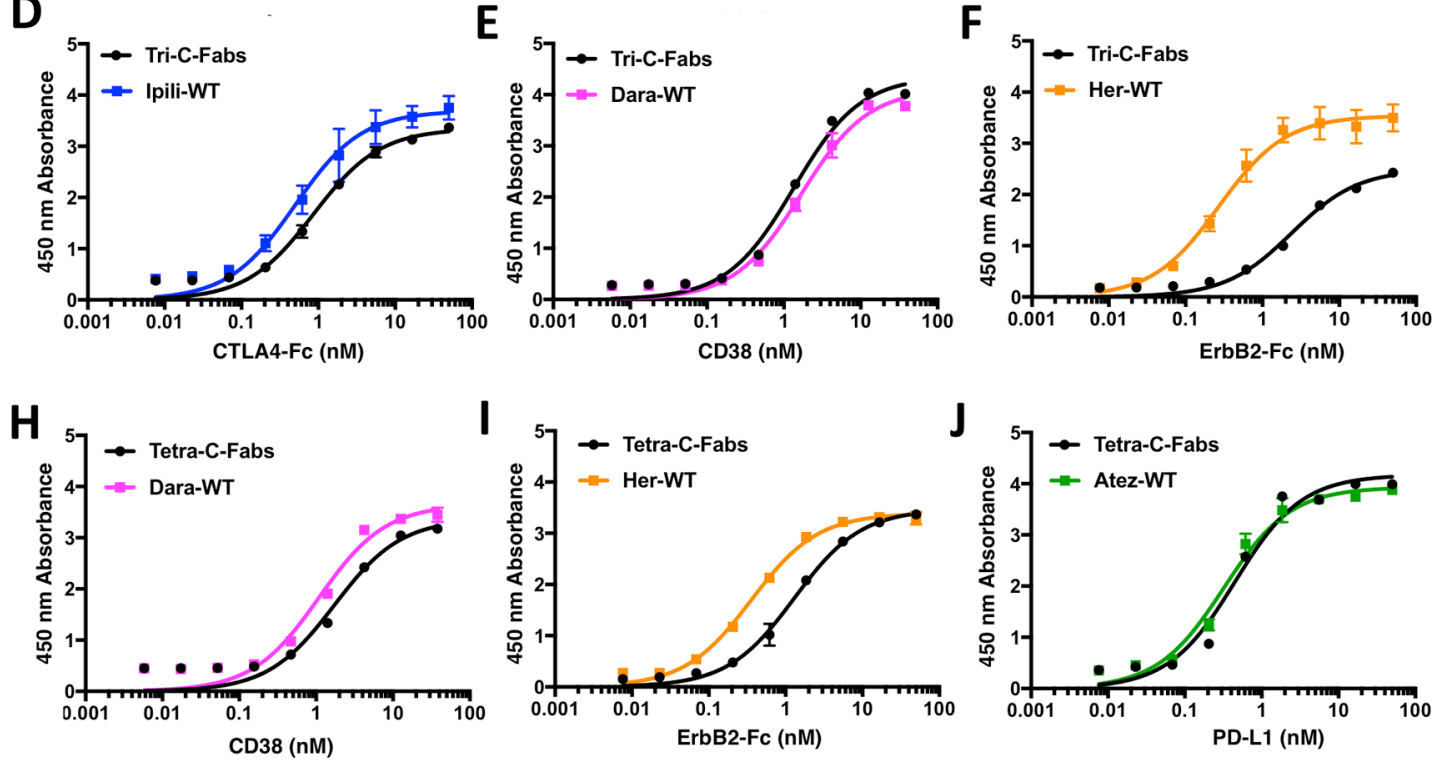

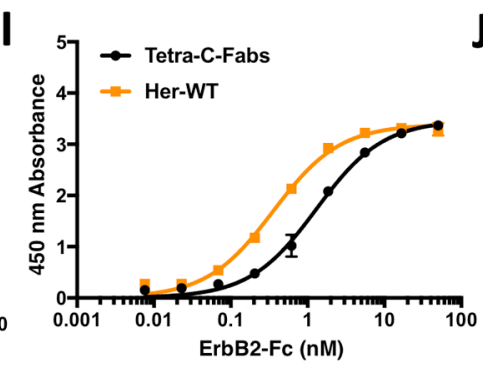

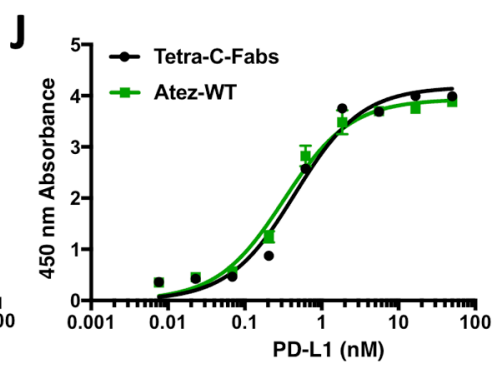

